\title{
UNIPOTENT ELEMENTS IN SMALL CHARACTERISTIC, II
}

\author{
G. LusztiG
}

\section{INTRODUCTION}

0.1. Let $\mathbf{k}$ be an algebraically closed field of characteristic exponent $p \geq 1$. This paper is a study of unipotent elements in a special orthogonal group $S O_{Q}$ (where $Q$ is a nondegenerate quadratic form on a finite dimensional $\mathbf{k}$-vector space $V$ ) with emphasis on the case where $p=2$. We develop some of the proposals in [L2] which try to extend the Dynkin-Kostant theory $[\mathrm{K}]$ of unipotent elements in the case $p=1$ to the case $p>1$.

Namely we show that to any unipotent element $u \in G$ one can associate canonically a filtration of $V$ whose is stabilizer in $S O_{Q}$ is a parabolic subgroup containing $u$ in the unipotent radical and such that this parabolic is of the same type as a parabolic attached in the Dynkin-Kostant theory to a unipotent element in a special orthogonal group with $p=1$. This allows us to partition the unipotent variety of $S O_{Q}$ into pieces which are both smooth varieties and unions of (possibly several) unipotent conjugacy classes. We show that (for a split rational structure over a finite field with $q$ elements) each piece has a number of rational points given by a polynomial in $q$ with integer coefficients independent of $q$ and $p$. (This kind of property was stated without proof in [L1].) The similar statement was established for groups of type $C$ in [L2]; for an exceptional type this is is easily established using the available tables since in that case each piece contains at most two unipotent conjugacy classes. In some sense the behaviour of the pieces is more complicated for orthogonal groups than for other almost simple groups. For example this is the only case where the number of unipotent conjugacy classes in a given piece (in bad characteristic) is not necessarily a power of 2 .

Notation. We set $\mathbf{Z}^{\prime}=2 \mathbf{Z}+1, \mathbf{Z}^{\prime \prime}=2 \mathbf{Z}$. For $a \in \mathbf{Z}$ we set $\mathbf{Z}_{\geq a}^{\prime}=\left\{n \in \mathbf{Z}^{\prime} ; n \geq\right.$ $a\}, \mathbf{Z}_{\geq a}^{\prime \prime}=\left\{n \in \mathbf{Z}^{\prime \prime} ; n \geq a\right\}$. The cardinal of a finite set $X$ is denoted by $|X|$; if $X$ is an infinite set we write $|X|=\infty$.

\section{Contents}

1. Unipotent elements in orthogonal groups.

2. The case $p=2$.

Supported in part by the National Science Foundation.

Typeset by $\mathcal{A} \mathcal{M} \mathcal{S}-\mathrm{T}_{\mathrm{E}} \mathrm{X}$ 
3. The case $p \neq 2$.

4. On unipotent conjugacy classes in $S O_{Q}(p=2)$.

\section{UNIPOTENT ELEMENTS IN ORTHOGONAL GROUPS}

1.1. Let $\mathbf{k}$ be a finite or algebraically closed field of characteristic exponent $p \geq 1$. Let $\mathcal{C}$ be the category whose objects are $\mathbf{k}$-vector spaces of finite dimension; the morphisms are linear maps. Let $\mathcal{C}^{\prime}$ be the subcategory of $\mathcal{V}$ consisting of all $V \in \mathcal{V}$ such that $\operatorname{dim} V \in \mathbf{Z}^{\prime}$. Let $\mathcal{C}^{\prime \prime}$ be the subcategory of $\mathcal{V}$ consisting of all $V \in \mathcal{V}$ such that $\operatorname{dim} V \in \mathbf{Z}^{\prime \prime}$. Let $\overline{\mathcal{C}}$ be the category whose objects are $\mathbf{Z}$-graded $\mathbf{k}$ vector spaces $\bar{V}=\oplus_{a \in \mathbf{Z}} \bar{V}^{a}$ such that $\operatorname{dim} \bar{V}<\infty$; the morphisms are linear maps respecting the grading.

Let $V \in \mathcal{C}$. For $N \in \operatorname{End} V$ nilpotent and $i \geq 1$ let

$E_{i}^{N}=\operatorname{ker} N^{i} /\left(N\left(\operatorname{ker} N^{i+1}\right)+\operatorname{ker} N^{i-1}\right)$.

Then $c_{i}^{N}:=\operatorname{dim} E_{i}^{N}$ is the number of Jordan blocks of size $i$ of $N$. Let $e=e_{N}$ be the smallest integer $\geq 0$ such that $N^{e}=0$.

For any symmetric or symplectic bilinear form $\langle\rangle:, V \times V \rightarrow \mathbf{k}$ let $\operatorname{Rad}_{\langle,\rangle}=$ $\{x \in V ;\langle x, V\rangle=0\}$ be the radical of $\langle$,$\rangle .$

In the remainder of this paper we fix $V \in \mathcal{C}$ and a quadratic form $Q: V \rightarrow \mathbf{k}$ with associated symmetric bilinear form $\langle\rangle:, V \times V \rightarrow \mathbf{k}$; we have $\langle x, y\rangle=$ $Q(x+y)-Q(x)-Q(y)$ for $x, y \in V$. Let $D=\operatorname{dim} V$. Let $R=\operatorname{Rad}_{\langle,\rangle}$. We assume that $Q$ is nondegenerate that is, $\left.Q\right|_{R}: R \rightarrow \mathbf{k}$ is injective. (In this case we must have $R=0$ unless $V \in \mathcal{C}^{\prime}, p=2$ when $\operatorname{dim} R=1$.)

In the case where $D \in \mathbf{Z}^{\prime \prime}$ let $\mathcal{J}_{Q}$ be the set of $D / 2$-dimensional subspaces of $V$ on which $Q$ is 0 . In the case where $|\mathbf{k}|<\infty, D \in \mathbf{Z}_{\geq 2}^{\prime \prime}$ we set $\eta_{Q}=1$ if $\mathcal{J}_{Q} \neq \emptyset$ and $\eta_{Q}=-1$ if $\mathcal{J}_{Q}=\emptyset$.

For any subspace $W$ of $V$ we set $W^{\perp}=\{x \in V ;\langle x, W\rangle=0\}$.

Let $O_{Q}=\{T \in G L(V) ; Q(T x)=Q(x) \quad \forall x \in V\}$ be the orthogonal group of $Q$. In the case where $|\mathbf{k}|=\infty$ we denote by $S O_{Q}$ the identity component of $O_{Q}$; in the case where $|\mathbf{k}|<\infty$ we set $S O_{Q}=O_{Q} \cap S O_{\tilde{Q}}$ where $\tilde{Q}$ is the quadratic form obtained from $Q$ by extension of scalars to an algebraic closure of $\mathbf{k}$. Let

$$
\begin{aligned}
\tilde{\mathcal{M}}_{Q} & =\left\{N \in \operatorname{End}(V) ; 1+N \text { unipotent in } O_{Q}\right\} \\
& =\{N \in \operatorname{End}(V) ; N \text { nilpotent }, Q(N x)=-\langle x, N x\rangle \text { for all } x \in V\} .
\end{aligned}
$$

Note that if $N \in \tilde{\mathcal{M}}_{Q}$ and $x, y \in V$ then

$$
\langle x, N y\rangle+\langle N x, y\rangle+\langle N x, N y\rangle=0
$$

In particular, for $N \in \tilde{\mathcal{M}}_{Q}$ we have $\langle x, N y\rangle=\left\langle N^{\dagger} x, y\right\rangle$ for $x, y \in V$, where $N^{\dagger}:=(1+N)^{-1}-1=-N+N^{2}-N^{3}+\cdots \in \tilde{\mathcal{M}}_{Q}$.

Let $N \in \tilde{\mathcal{M}}_{Q}$. We have

$$
N V \cap R=0 .
$$


If $x \in V$ and $N x \in R$ then $Q(N x)=-\langle x, N x\rangle=0$; since $Q: R \rightarrow \mathbf{k}$ is injective we see that $N x=0$. Thus (a) holds.

From the definitions we have for $i \geq 1$ :

(b) $\left(\operatorname{ker} N^{i}\right)^{\perp}=N^{i} V+R,\left(N^{i} V\right)^{\perp}=\operatorname{ker} N^{i}$.

In particular, $N R \subset R$. Since $\operatorname{dim} R \leq 1$, it follows that $N R=0$.

1.2. In this subsection we assume that $p>1$ and that $|\mathbf{k}|=q<\infty$. For any $m \in Z Z_{\geq 1}^{\prime}$ we set

$$
P_{m}=q^{1 / 4\left(m^{2}-2 m+1\right)}\left(q^{2}-1\right)\left(q^{4}-1\right) \ldots\left(q^{m-1}-1\right) .
$$

For any $m \in \mathbf{Z}_{\geq 2}^{\prime \prime}$ and $\delta \in\{1,-1\}$ we set

$$
P_{m}^{\delta}=q^{1 / 4\left(m^{2}-2 m\right)}\left(q^{2}-1\right)\left(q^{4}-1\right) \ldots\left(q^{m-2}-1\right)\left(q^{m / 2}-\delta\right) .
$$

Let $\mathcal{V}$ be a $\mathbf{k}$-vector space with a nondegenerate quadratic form $u: \mathcal{V} \rightarrow \mathbf{k}$. Let $():, \mathcal{V} \times \mathcal{V} \rightarrow \mathbf{k}$ be the associate symmetric bilinear form. Let $\mathcal{R}=\operatorname{Rad}_{(,)}$. Let $s=$ $\operatorname{dim} \mathcal{V}$. Let $k \in[0, s]$. Let $S_{k} \mathcal{V}$ be the set of $k$-dimensional subspaces $\mathcal{W}$ of $\mathcal{V}$ such that $\left.u\right|_{\mathcal{W}}$ is nondegenerate. If $k \in \mathbf{Z}_{\geq 2}^{\prime \prime}$ we have a partition $S_{k} \mathcal{V}=\sqcup_{\delta \in\{1,-1\}} S_{k} \mathcal{V}^{\delta}$ where $S_{k} \mathcal{V}^{\delta}=\left\{\mathcal{W} \in S_{k} \mathcal{V} ; \eta_{u_{\mathcal{W}}}=\delta\right\}$. If $s \in \mathbf{Z}^{\prime \prime}, k \in \mathbf{Z}^{\prime}$ we set $N_{s, k}^{\epsilon, *}=\left|S_{k} \mathcal{V}\right|$. If $s \in \mathbf{Z}^{\prime}, k \in \mathbf{Z}^{\prime}$, we set $N_{s, k}^{*, *}=\left|S_{k} \mathcal{V}\right|$. If $s \in \mathbf{Z}^{\prime \prime}, k \in \mathbf{Z}_{\geq 2}^{\prime \prime}$ and $\delta \in\{1,-1\}$, we set $N_{s, k}^{\epsilon, \delta}=\left|S_{k}^{\delta} \mathcal{V}\right|$. If $s \in \mathbf{Z}^{\prime}, k \in \mathbf{Z}_{\geq 2}^{\prime \prime}$ and $\delta \in\{1,-1\}$, we set $N_{s, k}^{*, \delta}=\left|S_{k}^{\delta} \mathcal{V}\right|$.

(i) If $s \in \mathbf{Z}_{\geq 2}^{\prime \prime}, k \in \mathbf{Z}^{\prime}, \epsilon=\eta_{u}$ then $N_{s, k}^{\epsilon, *}=P_{s}^{\epsilon} P_{k}^{-1} P_{t-k}^{-1}$.

If $p \neq 2$ this is obvious. Assume now that $p=2$. Let $\mathcal{R}^{\prime}$ be the radical of $\left.()\right|_{,\mathcal{W}}$ for $\mathcal{W} \in S_{k} \mathcal{V}$. Then $\mathcal{R}^{\prime}$ is one of the $q^{(s-2) / 2}\left(q^{s / 2}-\epsilon\right)$ lines in $\mathcal{V}$ on which $u$ is not identically 0 . Also $\mathcal{R}^{\prime \perp}$ is a hyperplane in $\mathcal{V}$ and $($, ) induces a nondegenerate symplectic form on $\mathcal{R}^{\prime \perp} / \mathcal{R}^{\prime}$. For each such $\mathcal{R}^{\prime}$, the number of $\mathcal{W} \in S_{k} \mathcal{V}$ such that $\mathcal{R}^{\prime} \subset \mathcal{W} \subset \mathcal{R}^{\prime \perp}$ is the number of $(k-1)$-dimensional subspaces of $\mathcal{R}^{\prime \perp} / \mathcal{R}^{\prime}$ on which $($,$) is nondegenerate hence it is P_{s-1} P_{k}^{-1} P_{s-k}^{-1}$. Hence $N_{s, k}^{\epsilon, *}=q^{(s-2) / 2}\left(q^{s / 2}-\right.$ є) $P_{s-1} P_{k}^{-1} P_{s-k}^{-1}$, as required.

(ii) If $s \in \mathbf{Z}_{\geq 2}^{\prime \prime}, k \in \mathbf{Z}_{\geq 2}^{\prime \prime}, k<s, \epsilon=\eta_{u}, \delta \in\{1,-1\}$, then

$$
N_{s, k}^{\epsilon, \delta}=2^{-1} P_{s}^{\epsilon}\left(P_{k}^{\delta}\right)^{-1}\left(P_{s-k}^{\epsilon \delta}\right)^{-1} \text {. }
$$

This is clear: the orthogonal group of $u$ acts transitively on $S_{k}^{\delta} \mathcal{V}$.

(iii) If $s \in \mathbf{Z}^{\prime}, k \in \mathbf{Z}^{\prime}, k<s$, then $N_{s, k}^{*, *}=2^{-1} \sum_{\delta \in\{1,-1\}} P_{s} P_{k}^{-1}\left(P_{s-k}^{\delta}\right)^{-1}$.

If $p \neq 2$ this is obvious. Assume now that $p=2$. We set $s=2 t+1, k=2 a+1$. Let $\mathcal{R}^{\prime}=\operatorname{Rad}_{(,) \mid \mathcal{W}}$ for $\mathcal{W} \in S_{k} \mathcal{V}$. Then $\mathcal{R}^{\prime}$ is one of the $q^{2 t}$ lines in $\mathcal{V}$ on which $u$ is not identically 0 . The number of $\mathcal{W} \in S_{k} \mathcal{V}$ such that the corresponding $\mathcal{R}^{\prime}$ is equal to $\mathcal{R}$ is the number of $2 a$-dimensional subspaces of $\mathcal{V} / \mathcal{R}$ on which $($,$) is$ nondegenerate that is $P_{s} P_{k}^{-1} P_{s-k+1}^{-1}$. Let $N^{\prime}$ be the number of $\mathcal{W} \in S_{k} \mathcal{V}$ such that the corresponding $\mathcal{R}^{\prime}$ is not equal to $\mathcal{R}$. Then $N^{\prime}=\left(q^{s-1}-1\right) N_{0}^{\prime}$ where $N_{0}^{\prime}$ is the number of $\mathcal{W}$ with prescribed $\mathcal{R}^{\prime} \neq \mathcal{R}$. To such $\mathcal{W}$ we associate the subspace $\mathcal{W}^{\prime}=\mathcal{W}+\mathcal{R}$ (of dimension $k+1$ ) of $\mathcal{R}^{\prime \perp}$ (of dimension $s-1$ ). Note that the number of possible such $\mathcal{W}^{\prime}$ is the number of $k$-1-dimensional subspaces of $\mathcal{R}^{\prime \perp} /\left(\mathcal{R}+\mathcal{R}^{\prime}\right)$ on which $($,$) is nondegenerate that is P_{s-2} P_{k}^{-1} P_{s-k-1}^{-1}$. For any $\mathcal{W}^{\prime}$ as above the number of $\mathcal{W}$ such that $\mathcal{R}^{\prime} \subset \mathcal{W} \subset \mathcal{W}^{\prime}$ and $\mathcal{W} \oplus \mathcal{R}=\mathcal{W}^{\prime}$ is the 
number of subspaces $\mathcal{X} \subset \mathcal{W}^{\prime} / \mathcal{R}^{\prime}$ such that $\mathcal{X} \oplus \mathcal{R}=\mathcal{W}^{\prime} / \mathcal{R}^{\prime}$ that is $q^{k-1}$. Thus,

$$
\begin{aligned}
& N^{\prime}=\left(q^{s-1}-1\right) q^{k-1} P_{s-2} P_{k}^{-1} P_{s-k-1}^{-1} \\
& =q^{2 a} q^{-t^{2}+(t-1)^{2}} q^{(t-a)^{2}-(t-a-1)^{2}}\left(q^{2 t-2 a}-1\right) P_{s} P_{k}^{-1} P_{s-k+1}^{-1}
\end{aligned}
$$

We see that

$$
\begin{aligned}
N_{s, k}^{*, *} & =P_{s} P_{k}^{-1} P_{s-k+1}^{-1}+q^{2 a} q^{-t^{2}+(t-1)^{2}} q^{(t-a)^{2}-(t-a-1)^{2}}\left(q^{2 t-2 a}-1\right) P_{s} P_{k}^{-1} P_{s-k+1}^{-1} \\
& =q^{2 t-2 a} P_{2 t+1} P_{2 a+1}^{-1} P_{2 t-2 a+1}^{-1}
\end{aligned}
$$

and (iii) follows.

(iv) If $s \in \mathbf{Z}^{\prime}, k \in \mathbf{Z}_{\geq 2}^{\prime \prime}, \delta \in\{1,-1\}$ then $N_{s, k}^{*, \delta}=2^{-1} P_{s}\left(P_{k}^{\delta}\right)^{-1} P_{s-k}^{-1}$.

This is clear: the orthogonal group of $u$ acts transitively on $S_{k}^{\delta} \mathcal{V}$.

We now consider a descending sequence $r_{0} \geq r_{2} \geq r_{4} \geq \ldots$ of integers such that $r_{0}=s$ and $r_{2 n}=0$ for large $n$. We denote by $\nu\left(r_{0}, r_{2}, r_{4}, \ldots\right)$ (if $\left.s \in \mathbf{Z}^{\prime}\right)$ or by $\nu^{\epsilon}\left(r_{0}, r_{2}, r_{4}, \ldots\right)$ (if $\left.s \in \mathbf{Z}_{\geq 2}^{\prime \prime}, \eta_{u}=\epsilon\right)$ the number of sequences $U_{0} \supset U_{2} \supset U_{4} \supset \ldots$ of subspaces of $\mathcal{V}$ such that for any $n \geq 0, \operatorname{dim} U_{2 n}=r_{2 n}$ and the quadratic form $\left.u\right|_{U_{2 n}}$ is nondegenerate. We show:

(a) Let $X=\nu\left(r_{0}, r_{2}, r_{4}, \ldots\right)$ if $r_{0} \in \mathbf{Z}^{\prime}$ and $X=\nu^{\epsilon}\left(r_{0}, r_{2}, r_{4}, \ldots\right)$ if $r_{0} \in \mathbf{Z}_{\geq 2}^{\prime \prime}$ and $\epsilon \in\{1,-1\}$. Then $X$ is a polynomial in $q$ with coefficients in $\mathbf{Z}$ independent of $q$ or $p$.

Let $M$ be the number of nonzero terms in the sequence $\left(r_{0}, r_{2}, r_{4}, \ldots\right)$. We have $M \geq 1$. We argue by induction on $M$. If $M=1$ we have $X=1$. Assume now that $M \geq 2$. If $r_{2 n}=r_{2 n+2}>0$ for some $n$ we have $X=\nu\left(r_{0}, \ldots, r_{2 n}, r_{2 n+4}, \ldots\right)$ if $r_{0} \in \mathbf{Z}^{\prime}, X=\nu^{\epsilon}\left(r_{0}, \ldots, r_{2 n}, r_{2 n+4}, \ldots\right)$ if $r_{0} \in \mathbf{Z}^{\prime \prime}$; the result follows. Hence we may assume that the nonzero terms of $r_{0}, r_{2}, \ldots$ are distinct. If $r_{2 n} \in \mathbf{Z}^{\prime}$ for some $n>0$, we have $X=\nu\left(r_{0}, \ldots, r_{2 n}, 0,0,\right) \nu\left(r_{2 n}, r_{2 n+2}, \ldots\right)$ if $r_{0} \in \mathbf{Z}^{\prime}$, $X=\nu^{\epsilon}\left(r_{0}, \ldots, r_{2 n}, 0,0,\right) \nu\left(r_{2 n}, r_{2 n+2}, \ldots\right)$ if $r_{0} \in \mathbf{Z}^{\prime \prime}$; in both cases the induction hypothesis applies to the second factor in the right hand side. Thus we may assume that any odd number in the sequence $r_{0}, r_{2}, \ldots$ must appear either as $r_{0}$ or it must be followed by 0 . Thus we must consider four cases.

Case 1. $r_{2 n} \in \mathbf{Z}^{\prime \prime}$ for all $n$. Let $r_{2 m}$ be the last nonzero term of $r_{0}, r_{2}, \ldots$ If $m=0$ we have $X=1$. If $m>0$ we have, using (ii):

$$
\begin{aligned}
& X=\sum_{\delta_{1}, \delta_{2}, \ldots, \delta_{m} \in\{1,-1\}} N_{r_{0}, r_{2}}^{\epsilon, \delta_{1}} N_{r_{2}, r_{4}}^{\delta_{1}, \delta_{2}} \ldots N_{r_{2 m-2}, r_{2 m}}^{\delta_{m-1}, \delta_{m}} \\
& =2^{-m} \sum_{\delta_{1}, \delta_{2}, \ldots, \delta_{m} \in\{1,-1\}} P_{r_{0}}^{\epsilon}\left(P_{r_{0}-r_{2}}^{\epsilon \delta_{1}}\right)^{-1}\left(P_{r_{2}-r_{4}}^{\delta_{1} \delta_{2}}\right)^{-1} \ldots\left(P_{r_{2 m-2}-r_{2 m}}^{\delta_{m-1} \delta_{m}}\right)^{-1}\left(P_{r_{2 m}}^{\delta_{m}}\right)^{-1} .
\end{aligned}
$$

This is clearly a polynomial in $q$ with coefficients in $\mathbf{Z}\left[2^{-1}\right]$. It is also the product 
of a polynomial in $q$ with coefficients in $\mathbf{Z}$ with

$$
\begin{aligned}
& 2^{-m} \sum_{\delta_{1}, \delta_{2}, \ldots, \delta_{m} \in\{1,-1\}}\left(q^{\left(r_{0}-r_{2}\right) / 2}-\epsilon \delta_{1}\right)^{-1}\left(q^{\left(r_{2}-r_{4}\right) / 2}-\delta_{1} \delta_{2}\right)^{-1} \ldots \times \\
& \times\left(q^{\left(r_{2 m-2}-r_{2 m}\right) / 2}-\delta_{m-1} \delta_{m}\right)^{-1}\left(q^{r_{2 m} / 2}-\delta_{m}\right)^{-1} \\
& =\left(q^{r_{0} / 2}+\epsilon\right)\left(q^{r_{0}-r_{2}}-1\right)^{-1}\left(q^{r_{2}-r_{4}}-1\right)^{-1} \ldots\left(q^{r_{2 m-2}-r_{2 m}}-1\right)^{-1}\left(q^{r_{2 m}}-1\right)^{-1}
\end{aligned}
$$

hence is a power series with integer coefficients in $q$. Hence $X \in \mathbf{Z}[q]$.

Case 2. $r_{0} \in \mathbf{Z}^{\prime \prime}, r_{2 m} \in \mathbf{Z}^{\prime}, r_{2 n} \in \mathbf{Z}^{\prime \prime}$ for $0<n<m$ and $r_{2 n}=0$ for $n>m$. If $m=1$ we have $X=N_{r_{0}, r_{2}}^{\epsilon, *}$. If $m>1$ we have

$$
X=\sum_{\delta_{1}, \delta_{2}, \ldots, \delta_{m-1} \in\{1,-1\}} N_{r_{0}, r_{2}}^{\epsilon, \delta_{1}} N_{r_{2}, r_{4}}^{\delta_{1}, \delta_{2}} \ldots N_{r_{2 m-4}, r_{2 m-2}}^{\delta_{m-2}, \delta_{m-1}} N_{r_{2 m-2}, r_{2 m}}^{\delta_{m-1}, *}
$$

As in case 1 we see, using (ii) and (i) that $X \in \mathbf{Z}[q]$.

Case 3. $r_{0} \in \mathbf{Z}^{\prime}, r_{2 n} \in \mathbf{Z}^{\prime \prime}$ for $n>0$. Let $r_{2 m}$ be the last nonzero term of $r_{0}, r_{2}, \ldots$ We have

$$
X=\sum_{\delta_{1}, \delta_{2}, \ldots, \delta_{m} \in\{1,-1\}} N_{r_{0}, r_{2}}^{*, \delta_{1}} N_{r_{2}, r_{4}}^{\delta_{1}, \delta_{2}} \ldots N_{r_{2 m-2}, r_{2 m}}^{\delta_{m-1}, \delta_{m}}
$$

As in case 1 we see, using (ii) and (iv) that $X \in \mathbf{Z}[q]$.

Case 4. $r_{0} \in \mathbf{Z}^{\prime}$ and for some $m>0, r_{2 m} \in \mathbf{Z}^{\prime}, r_{2 n} \in \mathbf{Z}^{\prime \prime}$ for $0<n<m$ and $r_{2 n}=0$ for $n>m$. If $m=1$ we have $X=N_{r_{0}, r_{2}}^{*, *}$, see (iii). If $m>1$ we have

$$
X=\sum_{\delta_{1}, \delta_{2}, \ldots, \delta_{m-1} \in\{1,-1\}} N_{r_{0}, r_{2}}^{*, \delta_{1}} N_{r_{2}, r_{4}}^{\delta_{1}, \delta_{2}} \ldots N_{r_{2 m-4}, r_{2 m-2}}^{\delta_{m-2}, \delta_{m-1}} N_{r_{2 m-2}, r_{2 m}}^{\delta_{m-1}, *}
$$

As in case 1 we see, using (i), (ii) and (iv) that $X \in \mathbf{Z}[q]$. This completes the proof of (a).

For any $m \in \mathbf{Z}_{\geq 0}^{\prime \prime}$ we set

$$
R_{m}=q^{\left.m^{2} / 4\right)}\left(q^{2}-1\right)\left(q^{4}-1\right) \ldots\left(q^{m}-1\right) .
$$

Let $\mathcal{V}^{\prime}$ be a $\mathbf{k}$-vector space with a nondegenerate symplectic form $():, \mathcal{V}^{\prime} \times \mathcal{V}^{\prime} \rightarrow \mathbf{k}$.

We consider a descending sequence $r_{1} \geq r_{3} \geq r_{5} \geq \ldots$ of even integers such that $r_{1}=\operatorname{dim} \mathcal{V}^{\prime}$ and $r_{2 n+1}=0$ for large $n$. We denote by $\nu^{\prime}\left(r_{1}, r_{3}, r_{5}, \ldots\right)$ the number of sequences $U_{1} \supset U_{3} \supset U_{5} \supset \ldots$ of subspaces of $\mathcal{V}^{\prime}$ such that for any $n \geq 0, \operatorname{dim} U_{2 n+1}=r_{2 n+1}$ and the symplectic form $\left.()\right|_{,U_{2 n+1}}$ is nondegenerate. Clearly,

(b) $\nu^{\prime}\left(r_{1}, r_{3}, r_{5}, \ldots\right)=R_{r_{1}} R_{r_{1}-r_{3}}^{-1} R_{r_{3}-r_{5}}^{-1} \ldots$ is a polynomial in $q$ with coefficients in $\mathbf{Z}$ independent of $q$ or $p$. 
1.3. Let $\bar{V} \in \overline{\mathcal{C}}$. Define $\left(f_{a}\right)_{a \in \mathbf{Z}}$ by $f_{a}=\operatorname{dim} \bar{V}^{a}$. A quadratic form $\bar{Q}: \bar{V} \rightarrow \mathbf{k}$ with associated symmetric bilinear form $\langle$,$\rangle is said to be compatible with the$ grading if $\left.\bar{Q}\right|_{\bar{V}^{a}}=0$ for $a \neq 0$ and $\left\langle\bar{V}^{a}, \bar{V}^{a^{\prime}}\right\rangle=0$ for $a+a^{\prime} \neq 0$. In this subsection we fix such a $\bar{Q}$ (compatible with the grading) and we assume that it is nondegenerate. Let $\bar{R}=\operatorname{Rad}_{\langle,\rangle}$. Then $\bar{R} \subset \bar{V}^{0},\left.Q\right|_{\bar{V}^{0}}: \bar{V}^{0} \rightarrow \mathbf{k}$ is nondegenerate and $\langle$,$\rangle restricts$ to a perfect pairing $\bar{V}^{-a} \times \bar{V}^{a} \rightarrow \mathbf{k}$ for any $a \geq 1$. Hence $f_{a}=f_{-a}$ for all $a$. Let

$$
\begin{aligned}
E^{2} \bar{V} & =\left\{T \in \operatorname{Hom}(\bar{V}, \bar{V}) ; T\left(\bar{V}^{a}\right) \subset \bar{V}^{a+2} \text { for any } a \in \mathbf{Z} ;\langle T x, y\rangle\right. \\
& \left.+\langle x, T y\rangle=0 \text { for all } x, y \in \bar{V} ;\langle x, T x\rangle=0 \text { for any } x \in \bar{V}^{-1}\right\} .
\end{aligned}
$$

If $T \in E^{2} \bar{V}$ we have

(a) $\left\langle x, T^{a} x\right\rangle=0$ for any $a \in Z Z_{\geq 1}^{\prime}$ and any $x \in \bar{V}^{-a}$.

Indeed, we have $a=2 a^{\prime}+1$ with $a^{\prime} \geq 0$ and $x^{\prime}=T^{a^{\prime}} x \in \bar{V}^{-1}$ satisfies $\left\langle x^{\prime}, T x^{\prime}\right\rangle=0$. Thus

$$
0=\left\langle T^{a^{\prime}} x, T^{a^{\prime}+1} x\right\rangle=(-1)^{a^{\prime}}\left\langle x, T^{a}\right\rangle
$$

and (a) follows.

Let $E_{*}^{2} \bar{V}$ be the set of all $T \in E^{2} \bar{V}$ such that

(i) for any $a \in \mathbf{Z}_{\geq 0}^{\prime \prime}$, the quadratic form $\bar{Q}_{a}: \bar{V}^{-a} \rightarrow \mathbf{k}, x \mapsto \bar{Q}\left(T^{a / 2} x\right)$ is nondegenerate;

(ii) for any $a \in Z Z_{\geq 1}^{\prime}$, the symplectic form $\bar{V}^{-a} \times \bar{V}^{-a} \rightarrow \mathbf{k}, x, y \mapsto\left\langle x, T^{a} y\right\rangle$ is nondegenerate.

Note that (i) is automatic for $a=0$.

For $\tilde{N} \in E^{2} \bar{V}$ and $a \geq 0$ we set $K_{a}^{T}=\operatorname{ker}\left(T^{a}: \bar{V}^{-a} \rightarrow \bar{V}^{a}\right)$. If $a \geq 1$ then $K_{a}^{T}=\operatorname{Rad}_{\langle,\rangle_{a}}$ where $\langle,\rangle_{a}: \bar{V}^{-a} \times \bar{V}^{-a} \rightarrow \mathbf{k}$ is $x, y \mapsto\left\langle x, T^{a} y\right\rangle$. Hence condition (ii) is equivalent to the condition that $K_{a}^{T}=0$ for $a \in Z Z_{\geq 1}^{\prime}$. If $a \in \mathbf{Z}_{\geq 0}^{\prime \prime}$ then the symmetric bilinear form associated to $\bar{Q}_{a}$ is $(-1)^{a / 2}\langle,\rangle_{a}$. Hence condition (i) is equivalent to the condition that $\bar{Q}_{a}: K_{a}^{T} \rightarrow \mathbf{k}$ is injective for $a \in \mathbf{Z}_{>2}^{\prime \prime}$. (It implies that for such $a$ we have $K_{a}^{T}=0$ unless $p=2$ and $f_{-a} \in \mathbf{Z}^{\prime}$, when $\operatorname{dim} K_{a}^{T}=1$.)

This discussion shows that if $p \neq 2$ an element $T \in E^{2} \bar{V}$ belongs to $E_{*}^{2} \bar{V}$ if and only if $T^{a}: \bar{V}^{-a} \rightarrow \bar{V}^{a}$ is an isomorphism for any $a \geq 0$.

Returning to the general case we reformulate the conditions (i),(ii) for an element $T \in E^{2} \bar{V}$ to be in $E_{*}^{2} \bar{V}$ as follows:

(i') for any $a \in \mathbf{Z}_{\geq 0}^{\prime \prime}$, the map $T^{a / 2}: \bar{V}^{-a} \rightarrow \bar{V}^{0}$ is injective and its image $I_{a}^{T}$ is such that $\left.\bar{Q}\right|_{I_{a}^{T}}$ is a nondegenerate quadratic form;

(ii') the symplectic form $\omega_{T}(x, y)=\langle x, T y\rangle$ on $V^{-1}$ is nondegenerate; for any $a \in Z Z_{\geq 1}^{\prime}$, the map $T^{(a-1) / 2}: \bar{V}^{-a} \rightarrow \bar{V}^{-1}$ is injective and its image $I_{a}^{T}$ is such that $\left.\omega_{T}\right|_{I_{a}^{T}}$ is a nondegenerate symplectic form.

Clearly if (i') holds then (i) holds. If (i) holds and $x \in \operatorname{ker}\left(T^{a / 2}: \bar{V}^{-a} \rightarrow \bar{V}^{0}\right)$ (with $a \in \mathbf{Z}_{\geq 2}^{\prime \prime}$ ) then $T^{a} x=0$ hence $x \in K_{a}^{T}$. We have also $\bar{Q}_{a}(x)=0$. Since $\bar{Q}_{a}: K_{a}^{T} \rightarrow \mathbf{k}$ is injective we see that $x=0$. We see that (i') holds.

For $a \in Z Z_{\geq 1}^{\prime}$ and $x, y \in \bar{V}^{-a}$ we have 


$$
\langle x, y\rangle_{a}=(-1)^{(a-1) / 2}\left\langle T^{(a-1) / 2} x, T^{(a-1) / 2} y\right\rangle_{1} .
$$

Hence if (ii') holds then (ii) holds. If (ii) holds and $x \in \operatorname{ker}\left(T^{(a-1) / 2}: \bar{V}^{-a} \rightarrow \bar{V}^{-1}\right.$ ) (with $a \in Z Z_{\geq 1}^{\prime}$ ) then $T^{a} x=0$ hence $x \in K_{a}^{T}$ and $x=0$. We see that (ii') holds.

If $T \in E_{*}^{2} \bar{V}$ then clearly,

$$
\bar{V}^{0}=I_{0}^{T} \supset I_{2}^{T} \supset I_{4}^{T} \supset \ldots \text { and } \bar{V}^{-1}=I_{1}^{T} \supset I_{3}^{T} \supset I_{5}^{T} \supset \ldots
$$

We see that if $E_{*}^{2} \bar{V} \neq \emptyset$ then

(b) $f_{0} \geq f_{-2} \geq f_{-4} \geq \ldots$ and $f_{-1} \geq f_{-3} \geq f_{-5} \geq \ldots$,

(c) $f_{a} \in \mathbf{Z}^{\prime \prime}$ if $a \in \mathbf{Z}^{\prime}$.

We say that $\left(f_{a}\right)_{a \in \mathbf{Z}}$ is admissible if it satisfies (b),(c). In the remainder of this subsection we assume that $\left(f_{a}\right)_{a \in \mathbf{Z}}$ is admissible. Let $R^{0}$ be the set of all sequences $U_{0} \supset U_{2} \supset U_{4} \supset \ldots$ of subspaces of $\bar{V}^{0}$ such that $\operatorname{dim} U_{a}=f_{-a}$ and $\left.\bar{Q}\right|_{U_{a}}$ is a nondegenerate quadratic form for $a=0,2,4, \ldots$ Let $R^{-1}$ be the set of all pairs $\left(\omega,\left(U_{1}, U_{3}, U_{5}, \ldots\right)\right)$ where $\omega$ is a nondegenerate symplectic form on $\bar{V}^{-1}$ and $U_{1} \supset U_{3} \supset U_{5} \supset \ldots$ are subspaces of $\bar{V}^{-1}$ such that $\operatorname{dim} U_{a}=f_{-a}$ and $\left.\omega\right|_{U_{a}}$ is a nondegenerate symplectic form for $a=1,3,5, \ldots$ Clearly $R^{0} \neq \emptyset, R^{-1} \neq \emptyset$. Define $\psi: E_{*}^{2} \bar{V} \rightarrow R^{0} \times R^{-1}$ by

$$
T \mapsto\left(\left(I_{0}^{T}, I_{2}^{T}, I_{4}^{T}, \ldots\right),\left(\omega_{T},\left(I_{1}^{T}, I_{3}^{T}, I_{5}^{T}, \ldots\right)\right)\right) .
$$

Clearly,

(d) the fibre of $\psi$ at $\left(\left(U_{0}, U_{2}, U_{4}, \ldots\right),\left(\omega,\left(U_{1}, U_{3}, U_{5}, \ldots\right)\right)\right) \in R^{0} \times R^{-1}$ can be identified with $\prod_{a \geq 2} \operatorname{Iso}\left(\bar{V}^{-a}, U_{a}\right)$;

here $\operatorname{Iso}\left(\bar{V}^{-a}, U_{a}\right)$ is the set of vector space isomorphisms $\bar{V}^{-a} \rightarrow U_{a}$.

We now assume that $\mathbf{k}, q$ are as in 1.2 . For any $m \geq 0$ we set

$A_{m}=q^{1 / 2\left(n^{2}-n\right)}(q-1)\left(q^{2}-1\right) \ldots\left(q^{m}-1\right)$.

If $f_{0} \in \mathbf{Z}_{\geq 2}^{\prime \prime}$ let $\epsilon=\eta_{\left.\bar{Q}\right|_{\bar{V} 0}}$. From (d) we see that

(e) $\left|E_{*}^{2} \bar{V}\right|=\prod_{a \geq 1} A_{f_{a}} \xi \nu^{\prime}\left(f_{1}, f_{3}, f_{5}, \ldots\right)\left(R_{f_{1}}\right)^{-1}$

where $\xi=\nu\left(f_{0}, f_{2}, f_{4}, \ldots\right)$ if $f_{0} \in \mathbf{Z}^{\prime} ; \xi=\nu^{\epsilon}\left(f_{0}, f_{2}, f_{4}, \ldots\right)$ if $f_{0} \in \mathbf{Z}_{\geq 2}^{\prime \prime} ; \xi=1$ if $f_{0}=0$.

1.4. Let $X^{*}=\left(X^{\geq a}\right)_{a \in \mathbf{Z}}$ be a sequence of subspaces of $V$ such that $X^{\geq a+1} \subset X^{\geq a}$ for any $a, X^{\geq a}=0$ for some $a, X^{\geq a}=V$ for some $a$ that is a filtration (see [L2, 2.2]) of $V$. We say that $X^{*}$ is a $Q$-filtration of $V$ if for any $a \geq 1$ we have

(a) $\left.Q\right|_{X \geq a}=0$ and $X^{\geq 1-a}=\left(X^{\geq a}\right)^{\perp}$.

Then for any $a \leq 0$ we have:

(b) $\left(X^{\geq a}\right)^{\perp}=X^{\geq 1-a}+R$ (direct sum) and $X^{\geq 1-a}=\left(X^{\geq a}\right)^{\perp} \cap Q^{-1}(0)$.

The first equality follows by applying $\perp^{\perp}$ to both sides of $X^{\geq a}=\left(X^{\geq 1-a}\right)^{\perp}$, see (a). If $x \in V^{\geq 1-a} \cap R$ then by (a) we have $Q(x)=0$. Since $Q: R \rightarrow \mathbf{k}$ is injective, we have $x=0$. Thus, $V^{\geq 1-a} \cap R=0$. The second equality in (b) follows from the first equality and (a). This proves (b).

The proof of the following result is standard.

(c) We can find a direct sum decomposition $V=\oplus_{a \in \mathbf{Z}} X^{a}$ such that $X^{\geq a}=$ $\oplus_{a^{\prime} ; a^{\prime} \geq a} X^{a}$ for all $a$ and $\left\langle X^{a}, X^{a^{\prime}}\right\rangle=0$ for $a+a^{\prime} \neq 0,\left.Q\right|_{X^{a}}=0$ for $a \neq 0$.

For any $Q$-filtration $X^{*}$ of $V$ we set

$$
E^{\geq 2} X^{*}=\left\{N \in \tilde{\mathcal{M}}_{Q} ; N X^{\geq a} \subset X^{\geq a+2} \text { for all } a \in \mathbf{Z}\right\} .
$$


We show:

(d) If $x \in X^{\geq-1}$ then $\langle x, N x\rangle=0$.

It is enough to show that $Q(N x)=0$. Since $N x \in X^{\geq 1}$, this follows from (a).

1.5. Let $X^{*}$ be a $Q$-filtration of $V$. We set $\operatorname{gr}^{a} X^{*}=X^{\geq a} / X^{\geq a+1}$. Then $\operatorname{gr} X^{*}=$ $\oplus_{a \in \mathbf{Z} g r}{ }^{a} X^{*} \in \overline{\mathcal{C}}$. Let $f_{a}=\operatorname{dimgr}^{a} X^{*}$. Define a quadratic form $\bar{Q}: \operatorname{gr} X^{*} \rightarrow \mathbf{k}$ by $\bar{Q}(x)=Q\left(\dot{x}_{0}\right)+\sum_{a \geq 1}\left\langle\dot{x}_{-a}, \dot{x}_{a}\right\rangle$ where $x=\sum_{a} x_{a}$ (with $x_{a} \in \operatorname{gr}^{a} X^{*}$ ) and $\dot{x}_{b}$ is a representative of $x_{b}$ in $X^{\geq b}$. The symmetric bilinear form associated to $\bar{Q}$ is $\sum_{a} x_{a}, \sum_{b} x_{b}^{\prime} \mapsto \sum_{a+b=0}\left\langle\dot{x}_{a}, \dot{x}_{b}^{\prime}\right\rangle$ where $x_{a} \in \operatorname{gr}^{a} X^{*}, x_{b}^{\prime} \in \operatorname{gr}^{b} X^{*}$ and $\dot{x}_{a}, \dot{x}_{b}^{\prime}$ are representatives of $x_{a}, x_{b}^{\prime}$ in $X^{\geq a}, X^{\geq b}$. This form is denoted again by $\langle$,$\rangle ; its$ radical is the image of $R$ under $X^{\geq 0} \rightarrow \operatorname{gr}^{0} X^{*}$. It follows that $\bar{Q}$ is nondegenerate. It is clearly compatible with the grading. Hence $f_{a}=f_{-a}$ for any $a$.

Now let $N \in E^{\geq 2} X^{*}$. For any $a, N$ restricts to a linear map $X^{\geq a} \rightarrow X^{\geq a+2}$ and $X^{\geq a+1} \rightarrow X^{\geq a+3}$ hence it induces a linear map $\operatorname{gr}^{a} X^{*} \rightarrow \operatorname{gr}^{a+2} X^{*}$. Taking the direct sum over $a$ of these linear maps we obtain a linear map $\bar{N}: \operatorname{gr} X^{*} \rightarrow \operatorname{gr} X^{*}$. We have $\bar{N} \in E^{2} \operatorname{gr} X^{*}$. (If $x \in \operatorname{gr}^{a} X^{*}, y \in \operatorname{gr}^{b} X^{*}$ and $\dot{x}, \dot{y}$ are representatives for $x, y$ in $X^{\geq a}, X^{\geq b}$, the sum $\langle\bar{N} x, y\rangle+\langle x+\bar{N} y\rangle$ is 0 unless $a+b+2=0$ in which case it $\langle N \dot{x}, \dot{y}\rangle+\langle\dot{x}+N \dot{y}\rangle=-\langle N \dot{x}, N \dot{y}\rangle$ which is again zero since $N \dot{x} \in X^{\geq a+2}$, $N \dot{y} \in X^{\geq b+2}=X^{\geq-a}$ and $\left\langle X^{\geq a+2}, X^{\geq-a}\right\rangle=0$. If $x, d x$ are as above and $a=-1$, we have $\langle x, \bar{N} x\rangle=\langle\dot{x}, N \dot{x}\rangle=-Q(N \dot{x})$ and this is 0 since $N \dot{x} \in X^{\geq 1}$ and $\left.Q\right|_{X \geq 1}=$ 0.) Thus we have a well defined map

$$
\Phi: E^{\geq 2} X^{*} \rightarrow E^{2} \operatorname{gr} X^{*}, \quad N \mapsto \bar{N}
$$

Let

$$
d=\sum_{a<a^{\prime} ;-a-a^{\prime} \geq 3} f_{a} f_{a^{\prime}}+\sum_{a ;-2 a \geq 4} f_{a}\left(f_{a}-1\right) / 2 .
$$

We show:

(a) $\Phi$ is an iterated affine space bundle with fibres of dimension d.

Let $V=\oplus_{a} X^{a}$ be as in 1.4(c). For any integer $k \geq 2$ let $Z_{k}$ be the set of all collections $\left(N_{b}^{a}\right)_{a, b \in \mathbf{Z} ; 2 \leq b-a \leq k}$ where $N_{b}^{a}: X^{a} \rightarrow X^{b}$ are linear maps that satisfy

(i) $\left\langle x_{a}, N_{-a}^{a^{\prime}} x_{a^{\prime}}^{\prime}\right\rangle+\left\langle N_{-a^{\prime}}^{a} x_{a}, x_{a^{\prime}}^{\prime}\right\rangle=-\sum_{b ;-a^{\prime}-2 \geq b \geq a+2}\left\langle N_{b}^{a} x_{a}, N_{-b}^{a^{\prime}} x_{a^{\prime}}^{\prime}\right\rangle$

for any $a \neq a^{\prime}$ such that $2 \leq-a-a^{\prime} \leq k$ and any $x_{a} \in X^{a}, x_{a^{\prime}}^{\prime} \in X^{a^{\prime}}$;

(ii) $\left\langle x_{a}, N_{-a}^{a} x_{a}\right\rangle=-Q\left(N_{0}^{a} x_{a}\right)-\sum_{b<0 ;-a-2 \geq b>a+2}\left\langle N_{b}^{a} x_{a}, N_{-b}^{a} x_{a}\right\rangle$

for any $a$ such that $4 \leq-2 a \leq k 0$ and any $x_{a} \in \bar{X}^{a}$;

(iii) $\left\langle x_{-1}, N_{1}^{-1} x_{-1}\right\rangle=0$

for any $x_{-1} \in X^{-1}$.

For large $k$ we may identify $Z_{k}=E^{\geq 2} X^{*}$ by $\left(N_{b}^{a}\right) \mapsto N, N x_{a}=\sum_{b ; b \geq a+2} N_{b}^{a} x_{a}$ with $x_{a} \in X^{a}$. Moreover we may identify $Z_{2}=E^{2} \operatorname{gr} X^{*}$ in an obvious way. We have obvious maps $Z_{2} \leftarrow Z_{3} \leftarrow Z_{4} \leftarrow \ldots$ These maps eventually become the identity map of $E^{\geq 2} X^{*}$; their composition may be identified with $\Phi$. It is enough to show that for any $k \geq 3$ the obvious map $Z_{k} \rightarrow Z_{k-1}$ is an affine space bundle 
with fibres of dimension

$$
d_{k}=\sum_{a<a^{\prime} ;-a-a^{\prime}=k} f_{a} f_{a^{\prime}}+\sum_{a ;-2 a=k} f_{a}\left(f_{a}-1\right) / 2
$$

We shall prove only that the fibre of this map at any given point of $Z_{k-1}$ is an affine space of dimension $d_{k}$. This fibre may be identified with the set of all collections $\left(N_{b}^{a}\right)_{a, b \in \mathbf{Z} ; b-a=k}$ where $N_{b}^{a}: X^{a} \rightarrow X^{b}$ are linear maps that satisfy (i) with $-a-a^{\prime}=k$, (ii) with $-2 a=k$. (In these equations the right hand sides involve only the coordinates of the given point in $Z_{k-1}$.) In the equation (i) with $a \neq a^{\prime},-a-a^{\prime}=k$ each of $N_{-a}^{a^{\prime}}, N_{-a^{\prime}}^{a}$ determines the other. So the solutions of this equation form an affine space of dimension $f_{a} f_{a^{\prime}}$. If $k \in \mathbf{Z}^{\prime}$ there are no further equations. If $k \in \mathbf{Z}^{\prime \prime}$, in the equation (ii) with $-2 a=k$ the right hand side is a known quadratic form on $X^{a}$ and the solutions $N_{-a}^{a}$ form an affine space of dimension $f_{a}\left(f_{a}-1\right) / 2$. This completes the proof of $(\mathrm{a})$.

We show:

(b) $1+E^{\geq 2} X^{*} \subset S O_{Q}$.

To prove this we may assume that $|\mathbf{k}|=\infty$. Since $1 \in 1+E^{\geq 2} X^{*} \subset O_{Q}$ it is enough to show that $E^{\geq 2} X^{*}$ is irreducible. Using (a) we see that it is enough to show that $E^{2} \operatorname{gr} X^{*}$ is irreducible. From the definitions we see that $E^{2} \operatorname{gr} X^{*}$ is an affine space of dimension $\sum_{a<a^{\prime} ;-a-a^{\prime}=2} f_{a} f_{a^{\prime}}+f_{-1}\left(f_{-1}-1\right) / 2$. This proves (b).

We set $E_{*}^{\geq 2} X^{*}=\Phi^{-1}\left(E_{*}^{2} \operatorname{gr} X^{*}\right)$. From (a) we see that:

(c) $E_{*}^{\geq 2} X^{*}$ is (via $\Phi$ ) an iterated affine space bundle over $E_{*}^{2} \operatorname{gr} X^{*}$ with fibres of dimension $d$.

Using this and results in 1.3 we see that if $E_{*}^{\geq 2} X^{*} \neq \emptyset$ then $\left(f_{a}\right)_{a \in \mathbf{Z}}$ is admissible.

In the remainder of this subsection we assume that $\mathbf{k}, q$ are as in 1.2 and that $\left(f_{a}\right)_{a \in \mathbf{Z}}$ is admissible. From (c) we see that

(d) $\left|E_{*}^{\geq 2} X^{*}\right|=q^{d}\left|E_{*}^{2} \operatorname{gr} X^{*}\right|$.

We denote $\left|E_{*}^{\geq 2} X^{*}\right|$ by $\mathfrak{B}_{\left(f_{a}\right)}$ if $D \in \mathbf{Z}^{\prime}$; by $\mathfrak{B}_{\left(f_{a}\right)}^{\epsilon}$ if $D \in \mathbf{Z}_{\geq 2}^{\prime \prime}$ and $\eta_{Q}=\epsilon$. From (d), 1.3(e), 1.2(a) and 1.2(b) we see that:

(e) Let $Y=\mathfrak{B}_{\left(f_{a}\right)}$ if $f_{0} \in \mathbf{Z}^{\prime} ; Y=\mathfrak{B}_{\left(f_{a}\right)}$ if $f_{0} \in \mathbf{Z}^{\prime \prime}, \sum_{a} f_{a}>0, \epsilon \in\{1,-1\}$. Then $Y$ is a polynomial in $q$ with coefficients in $\mathbf{Z}$ independent of $q$ or $p$.

Note that if $D \in \mathbf{Z}_{\geq 2}^{\prime \prime}$ we have $\eta_{Q}=\eta_{\bar{Q}}$.

1.6. In the remainder of this section we assume that $D \geq 2$. Let $\tilde{\mathcal{F}}^{D}$ be the set of all collections $\left(f_{a}\right)_{a \in \mathbf{Z}}$ of natural numbers such that $\sum_{a} f_{a}=D, f_{a}=f_{-a}$ for all $a$ and the admissibility conditions $1.3(\mathrm{~b}),(\mathrm{c})$ hold. When $D \in \mathbf{Z}^{\prime \prime}$, let $J$ be the set of $S O(V)$-orbits on $\mathcal{J}_{Q}$ (if $|\mathbf{k}|=\infty$ ) or on $\mathcal{J}_{\tilde{Q}}$ for $\tilde{Q}$ as in 1.1 (if $|\mathbf{k}|<\infty$ ); note that $|J|=2$; in this case let

$$
\begin{aligned}
& \tilde{\mathcal{F}}_{0}^{D}=\left\{\left(f_{a}\right) \in \tilde{\mathcal{F}}^{D} ; f_{0}=0\right\}, \tilde{\mathcal{F}}_{1}^{D}=\left\{\left(f_{a}\right) \in \tilde{\mathcal{F}}^{D} ; f_{0}>0\right\}, \\
& \mathcal{F}^{D}=\tilde{\mathcal{F}}_{1}^{D} \sqcup\left(J \times \tilde{\mathcal{F}}_{0}^{D}\right) .
\end{aligned}
$$

When $D \in \mathbf{Z}^{\prime}$ we set $\mathcal{F}^{D}=\tilde{\mathcal{F}}^{D}$. For $\phi \in \mathcal{F}^{D}$ of the form $\left(f_{a}\right)$ or $\left(j,\left(f_{a}\right)\right)$ (where 
$j \in J)$, let $\overline{\mathcal{Y}}_{\phi}$ be the set of all $Q$-filtrations $X^{*}$ of $V$ such that $\operatorname{dim} \operatorname{gr}^{a} X^{*}=f_{a}$ for all $a$ and (in the case where $\left.\phi=\left(j,\left(f_{a}\right)\right)\right), X^{0}=X^{1} \in j$; let $\mathcal{Y}_{\phi}$ be the set of all pairs $\left(X^{*}, N\right)$ such that $X^{*} \in \overline{\mathcal{Y}}_{\phi}$ and $N \in E_{*}^{\geq 2} X^{*}$. Note that $\overline{\mathcal{Y}}_{\phi}$ is a partial flag manifold of $S O_{Q}$. Moreover, the obvious map $\mathcal{Y}_{\phi} \rightarrow \overline{\mathcal{Y}}_{\phi}$ has fibres $E_{*}^{\geq 2} X^{*}$ which are smooth (if $|\mathbf{k}|=\infty$ ) since $E_{*}^{\geq 2} X^{*}$ is open in the affine space $E^{\geq 2} X^{*}$. We see that $\mathcal{Y}_{\phi}$ is naturally a smooth variety (if $|\mathbf{k}|=\infty$ ).

Define $\Psi: \sqcup_{\phi \in \mathcal{F} D} \mathcal{Y}_{\phi} \rightarrow\left\{g \in S O_{Q} ; g\right.$ unipotent $\}$ by $\left(X^{*}, N\right) \mapsto 1+N$ for $\left(X^{*}, N\right) \in \mathcal{Y}_{\phi}$. This is well defined by $1.5(\mathrm{~b})$.

Theorem 1.7. In the setup of 1.6, $\Psi$ is a bijection.

An equivalent statement is:

(a) For any unipotent element $g \in S O_{Q}$ there is a unique $Q$-filtration $X^{*}$ of $V$ such that $g-1 \in E_{*}^{\geq 2} X^{*}$.

It is enough to prove this in the case where $|\mathbf{k}|=\infty$. The proof is given in 2.12, 3.3 .

For any $\phi \in \mathcal{F}^{D}$ let $\Xi_{\phi}=\Psi\left(\mathcal{Y}_{\phi}\right)$. The theorem shows that the sets $\Xi_{\phi}$ form a partition of the variety of unipotent elements in $S O_{Q}$ and that for any $\phi \in \mathcal{F}^{D}$, $\Phi$ restricts to a bijection $\mathcal{Y}_{\phi} \rightarrow \Xi_{\phi}$. One can show that if $|\mathbf{k}|=\infty$ this is an isomorphism of the smooth variety $\mathcal{Y}_{\phi}$ with a subvariety of the variety of unipotent elements of $S O_{Q}$. Note that if $|\mathbf{k}|=\infty$ then each $\mathcal{Y}_{\phi}$ is nonempty hence each $\Xi_{\phi}$ is nonempty. (If $|\mathbf{k}|<\infty$ then $\Xi_{\phi}$ is nonempty unless $D \in \mathbf{Z}^{\prime \prime}, \eta_{Q}=-1, \phi \in J \times \tilde{\mathcal{F}}_{0}^{D}$ in which case it is empty.) In the case where $|\mathbf{k}|=\infty$ and $p \neq 2$, the subsets $\Xi_{\phi}$ are exactly the unipotent conjugacy classes in $S O_{Q}$. If $p=2$, the subsets $\Xi_{\phi}$ are unions of unipotent conjugacy classes in $S O_{Q}$ but not necessarily single conjugacy classes; see Section 4.

1.8. In the setup of 1.6 we assume that $\mathbf{k}, q$ are as in 1.2 . We assume that Theorem 1.7 holds. Let $\phi \in \mathcal{F}^{D}$. Assume that if $D \in \mathbf{Z}^{\prime \prime}, \eta_{Q}=-1$, then $\phi \in \tilde{\mathcal{F}}_{1}^{D}$. We have

(a) $\left|\mathcal{Y}_{\phi}\right|=\left|\overline{\mathcal{Y}}_{\phi}\right|\left|E_{*}^{\geq 2} X^{*}\right|$

for any $X^{*} \in \overline{\mathcal{Y}}_{\phi}$. We denote $\left|\mathcal{Y}_{\phi}\right|$ by $\tilde{\mathfrak{B}}_{\phi}$ if $D \in \mathbf{Z}^{\prime}$; by $\tilde{\mathfrak{B}}_{\phi}^{\epsilon}$ if $D \in \mathbf{Z}^{\prime \prime}$ and $\eta_{Q}=\epsilon$. From (a) and 1.5(e) we see that:

(b) Let $\tilde{Y}=\tilde{\mathfrak{B}}_{\phi}$ if $D \in \mathbf{Z}^{\prime} ; \tilde{Y}=\tilde{\mathfrak{B}}_{\phi}^{\epsilon}$ if $D \in \mathbf{Z}^{\prime \prime}$ and $\eta_{Q}=\epsilon$. Then $\tilde{Y}$ is a polynomial in $q$ with coefficients in $\mathbf{Z}$ independent of $q$ or $p$.

1.9. Let $N \in \tilde{\mathcal{M}}_{Q}-\{0\}, e=e_{N}$. We have $e \geq 2$. Let $\mathrm{E}$ be a complement to $\operatorname{ker} N^{e-1}$ in $V$. We show

(a) The $(-1)^{e-1}$-symmetric bilinear form $():, E \times E \rightarrow \mathbf{k}$ given by $(x, y)=$ $\left\langle x, N^{e-1} y\right\rangle$ is nondegenerate.

Assume that $v_{0} \in E,\left\langle v_{0}, N^{e-1} E\right\rangle=0$. Then $\left\langle N^{e-1} v_{0}, E\right\rangle=0$. Also,

$\left\langle N^{e-1} v_{0}, \operatorname{ker} N^{e-1}\right\rangle= \pm\left\langle v_{0}, N^{e-1} \operatorname{ker} N^{e-1}\right\rangle=0$.

Thus $\left\langle N^{e-1} v_{0}, E+\operatorname{ker} N^{e-1}\right\rangle=0$ that is $\left\langle N^{e-1} v_{0}, V\right\rangle=0$ hence $N^{e-1} v_{0} \in R$. Using 1.1(a) we deduce $N^{e-1} v_{0}=0$. We see that $v_{0} \in \operatorname{Ker} N^{e-1} \cap E=0$. This proves (a). 
We show:

(b) the linear map $E^{\oplus e} \rightarrow V$ given by $\left(v_{0}, v_{1}, \ldots, v_{e-1}\right) \mapsto v_{0}+N v_{1}+\cdots+$ $N^{e-1} v_{e-1}$ is injective and $\langle$,$\rangle is nondegenerate on its image W:=E+N E+\cdots+$ $N^{e-1} E$.

Assume that $v=v_{0}+N v_{1}+\cdots+N^{e-1} v_{e-1}$ with $v_{i} \in E$ satisfies $0=\langle v, E\rangle=$ $\langle v, N E\rangle=\cdots=\left\langle v, N^{e-1} E\right\rangle$. We must show that $v_{k}=0$ for $k \in[0, e-1]$. We argue by induction on $k$. Assume that $k \in[0, e-1]$ and that $v_{k^{\prime}}=0$ for $k^{\prime}<k$. From $0=\left\langle v, N^{e-1-k} E\right\rangle$ we get $\left\langle N^{k} v_{k}, N^{e-1-k} E\right\rangle=0$ hence $\left(v_{k}, E\right)=0$ hence $v_{k}=0$ (using (a)). This proves (b).

We show:

(c) we have $V=W \oplus Y$ (W as in (b), $\left.Y=W^{\perp}\right), R \subset Y$ and $Y$ is $N$-stable; moreover $N^{e-1} Y=0$.

The first assertion follows from (b). Now let $x \in Y$. We can write $x=x^{\prime}+x^{\prime \prime}$ where $x^{\prime} \in E, x^{\prime \prime} \in \operatorname{ker} N^{e-1}$. We have $N^{e-1} x=N^{e-1} x^{\prime} \in W \cap Y$ hence $N^{e-1} x=0$. This proves (c).

\section{THE CASE $p=2$}

2.1. In this section we assume that $p=2$. In this case the symmetric bilinear form $\langle$,$\rangle associated to Q$ is symplectic. Let $N \in \tilde{\mathcal{M}}_{Q}$.

For $i \geq 1$ we define $\lambda_{i}^{N}: \operatorname{ker} N^{i} \rightarrow \mathbf{k}$ by $x \mapsto\left\langle x, N^{i-1} x\right\rangle^{1 / 2}$. Note that $\lambda_{i}^{N}=0$ if $i \in \mathbf{Z}^{\prime}$ and $\lambda_{i}^{N}$ is linear for any $i$.

For $i \geq 1$ we define $\epsilon_{i}^{N} \in\{0,1\}$ by $\epsilon_{i}^{N}=0$ if $\lambda_{i}^{N}=0, \epsilon_{i}^{N}=1$ if $\lambda_{i}^{N} \neq 0$. Note that $\epsilon_{i}^{N}=0$ if $i \in \mathbf{Z}^{\prime}$.

2.2. Let $N \in \tilde{\mathcal{M}}_{Q}-\{0\}, e=e_{N}$. We have $e \geq 2$. Let $\lambda=\lambda_{e}^{N}$. Define a subspace $L=L_{N}$ of $V$ by

$$
\begin{aligned}
& L=N^{e-1} V \text { if } \lambda=0, \\
& L=(\operatorname{ker} \lambda)^{\perp} \text { if } \lambda \neq 0, R=0, \\
& L=\left\{x \in(\operatorname{ker} \lambda)^{\perp} ; q(x)=0\right\} \text { if } \lambda \neq 0, R \neq 0 .
\end{aligned}
$$

If $\lambda \neq 0$ then ker $\lambda$ is a hyperplane in $V$ containing $R$; thus $L$ is a line if $R=0$. If $\lambda \neq 0, R \neq 0$ then $K:=(\operatorname{ker} \lambda)^{\perp}$ is a two dimensional subspace containing $R$ so that $\langle$,$\rangle is zero on K$. Hence $q: K \rightarrow \mathbf{k}$ is a group homomorphism which restricts to a group isomorphism $R \rightarrow \mathbf{k}$ hence induces a group isomorphism $K / L \rightarrow \mathbf{k}$. We see that $K=L \oplus R$. Thus $L$ is a line complementary to $R$ in $K$ and $\operatorname{ker} \lambda=L^{\perp}$.

We show:

(a) $L \subset L^{\perp}$.

Assume first that $\lambda=0$. By 1.1(b) it is enough to show that $N^{e-1} V \subset \operatorname{ker} N^{e-1}$ which is clear since $2 e-2 \geq e$. If $\lambda \neq 0$ then (a) is clear since $L$ is a line.

We show:

(b) if $\lambda \neq 0$, then $L \subset N^{e-1}\left(L^{\perp}\right)$ if and only if $N^{e-1} V \in \mathcal{C}^{\prime \prime}$.

Define a symmetric bilinear form $($,$) on V$ by $\left(y, y^{\prime}\right)=\left\langle y, N^{e-1} y^{\prime}\right\rangle$. We have $\operatorname{Rad}_{(,)}=\left\{y^{\prime} \in V ; N^{e-1} y^{\prime} \in R\right\}=\operatorname{ker} N^{e-1}$ (since $N^{e-1} V \cap R=0$ ). We have $\lambda(y)=(y, y)^{1 / 2}$. Since $\left.\lambda\right|_{\operatorname{Rad}_{(,)}}=0$, we can find $v \in V$ such that $(v, y)=\lambda(y)$ that 
is $\left\langle N^{e-1} v, y\right\rangle=\lambda(y)$ for any $y \in V$. Since $\lambda \neq 0$ we have $N^{e-1} v \notin 0$. Clearly, if $y \in \operatorname{ker} \lambda$ then $\left\langle N^{e-1} v, y\right\rangle=0$. Thus $N^{e-1} v \in(\operatorname{ker} \lambda)^{\perp}$.

The symmetric bilinear form $(,)_{0}$ on $V$ given by $\left(y, y^{\prime}\right)_{0}=\left(y, y^{\prime}\right)+\lambda(y) \lambda\left(y^{\prime}\right)$ is symplectic: for $y \in V$ we have $(y, y)_{0}=(y, y)+\lambda(y)^{2}=0$. Since $\left.\lambda\right|_{\operatorname{ker} N^{e-1}}=0$ we see that $R_{0}:=\operatorname{Rad}_{(,)_{0}}$ contains $\operatorname{ker} N^{e-1}$. Since $(,)_{0}$ is symplectic we have $\operatorname{dim} R_{0}=D \bmod 2$.

Assume now that $N^{e-1} V \in \mathcal{C}^{\prime \prime}$. Then $\operatorname{dim} \operatorname{ker} N^{e-1}=D \bmod 2$. Hence $\operatorname{dim} \operatorname{ker} N^{e-1}=\operatorname{dim} R_{0} \bmod 2$ that is $R_{0} / \operatorname{ker} N^{e-1} \in \mathcal{C}^{\prime \prime}$. If $R_{0} \neq \operatorname{ker} N^{e-1}$ then $\operatorname{dim}\left(R_{0} / \operatorname{ker} N^{e-1}\right) \geq 2$. Hence the kernel of the linear map $R_{0} /$ ker $N^{e-1} \rightarrow \mathbf{k}$ induced by $\lambda: R_{0} \rightarrow \mathbf{k}$ is non-zero. Thus there exists $u \in R_{0}$ such that $u \notin \operatorname{ker} N^{e-1}$ and $\lambda(u)=0$. For any $y \in V$ we have $0=(y, u)_{0}=(y, u)+\lambda(u) \lambda(y)=(y, u)$ so that $u \in \operatorname{Rad}_{(,)}=\operatorname{ker} N^{e-1}$. This contradiction shows that $R_{0}=\operatorname{ker} N^{e-1}$. Since $N^{e-1} v \neq 0$ we have $v \notin \in \operatorname{ker} N^{e-1}$ hence $v \notin R_{0}$. Thus there exists $v^{\prime} \in V$ such that $\left(v, v^{\prime}\right)_{0} \neq 0$ that is, $\left(v, v^{\prime}\right)+(v, v)\left(v, v^{\prime}\right) \neq 0$. It follows that $1+(v, v) \neq 0$. Moreover we have $0=(v, v)_{0}=(v, v)+(v, v)(v, v)$ so that $(v, v)(1+(v, v))=0$. Since $1+(v, v) \neq 0$ we deduce that $(v, v)=0$ that is $\left\langle v, N^{e-1} v\right\rangle=0$. We have $Q\left(N^{e-1} v\right)=\left\langle N^{e-2} v, N^{e-1} v\right\rangle=\left\langle v, N^{2 e-3} v\right\rangle$. If $e \geq 3$ this is 0 since $2 e-3 \geq e$; if $e=2$ this is equals $\left\langle v, N^{e-1} v\right\rangle$ which is again 0 . We see that $N^{e-1} v$ is a non-zero vector in $\operatorname{ker}\left(Q:(\operatorname{ker} \lambda)^{\perp} \rightarrow \mathbf{k}\right)$. Hence $N^{e-1} v \in L-0$. Since $L$ is a line we see that $L$ is spanned by $N^{e-1} v$. To show that $L \subset N^{e-1}\left(L^{\perp}\right)$ it is enough to show that $v \in L^{\perp}$ or that $\langle v, L\rangle=0$ or that $\left\langle v, N^{e-1} v\right\rangle=0$. But this equality is already known. We see that $L \subset N^{e-1}\left(L^{\perp}\right)$.

Conversely, assume that $L \subset N^{e-1}\left(L^{\perp}\right)$. Let $x \in L-0$. We have $x=N^{e-1} t$ with $\langle t, L\rangle=0$. In particular, $\langle t, x\rangle=0$ that is $\lambda(t)=0$. Since $x \in L$ we have $z \in \operatorname{ker} \lambda \Longrightarrow\langle x, z\rangle=0$. Hence there exists $c \in \mathbf{k}$ such that $\langle x, z\rangle=c \lambda(z)$ for all $z \in V$. Since $x \notin R$ we have $c \neq 0$. Replacing $x$ by a non-zero scalar multiple we may assume that $\langle x, z\rangle=\lambda(z)$ that is $(t, z)=\lambda(z)$ for all $z \in V$. Let $R_{1}=\operatorname{Rad}_{\left.(,)\right|_{\text {ker } \lambda}}$. If $u \in R_{1}$ then we have $w \in \operatorname{ker} \lambda \Longrightarrow(u, w)=0$. Hence there exists $c^{\prime} \in \mathbf{k}$ such that $(u, w)=c^{\prime} \lambda(w)$ that is $(u, w)=c^{\prime}(t, w)$ for any $w \in V$. Hence $u-c^{\prime} t \in \operatorname{Rad}_{(,)}=\operatorname{ker} N^{e-1}$. We see that $R_{1} \subset \mathbf{k} t+\operatorname{ker} N^{e-1}$. The reverse inclusion is obvious. Thus, $R_{1}=\mathbf{k} t+\operatorname{ker} N^{e-1}$. We see that $($,$) induces$ a nondegenerate symmetric bilinear form on $\operatorname{ker} \lambda /\left(\mathbf{k} t+\operatorname{ker} N^{e-1}\right)$. This induced form is symplectic: the equality $\left(y, y^{\prime}\right)_{0}=\left(y, y^{\prime}\right)+\lambda(y) \lambda\left(y^{\prime}\right)$ with $(,)_{0}$ symplectic shows that $\left.()\right|_{,\operatorname{ker} \lambda}=\left.(,)_{0}\right|_{\operatorname{ker} \lambda}$. We deduce that $\operatorname{ker} \lambda /\left(\mathbf{k} t+\operatorname{ker} N^{e-1}\right) \in \mathcal{C}^{\prime \prime}$. Since $x \neq 0$ we have $t \notin \operatorname{ker} N^{e-1}$ hence $\operatorname{dim} \operatorname{ker} \lambda-1-\operatorname{dim} \operatorname{ker} N^{e-1} \in \mathbf{Z}^{\prime \prime}$ that is $D-2-\left(D-\operatorname{dim} N^{e-1} V\right) \in \mathbf{Z}^{\prime \prime}$. We see that $N^{e-1} V \in \mathcal{C}^{\prime \prime}$. This proves (b).

We show:

(c) We have $L \subset N^{e-1} V+R$. More precisely we have $L \subset N^{e-1} V$ except possibly in the case where $V \in \mathcal{C}^{\prime}, \lambda \neq 0, e=2, c_{e}^{N} \in \mathbf{Z}^{\prime}$.

If $\lambda=0$ this is obvious. Assume now that $\lambda \neq 0$. Since $\operatorname{ker} N^{e-1} \subset \operatorname{ker} \lambda$ we have $(\operatorname{ker} \lambda)^{\perp} \subset\left(\operatorname{ker} N^{e-1}\right)^{\perp}$ and using $1.1(\mathrm{~b}),(\operatorname{ker} \lambda)^{\perp} \subset N^{e-1} V+R$. Since $L \subset(\operatorname{ker} \lambda)^{\perp}$ we have $L \subset N^{e-1} V+R$. If $R=0$ then clearly $L \subset N^{e-1} V$. If $e \geq 3, R \neq 0$ we have $\left.Q\right|_{N^{e-1} V}=0$; indeed for $v \in V$ we have $Q\left(N^{e-1} v\right)=$ 
$\left\langle N^{e-2} v, N^{e-1} v\right\rangle=\left\langle v, N^{2 e-3} v\right\rangle=0$. Hence if $x \in L$ is written as $x=N^{e-1} v+v^{\prime}$ with $v \in V, v^{\prime} \in R$ then $0=Q(x)=Q\left(v^{\prime}\right)$. But $Q\left(v^{\prime}\right)=0, v^{\prime} \in R$ implies $v^{\prime}=0$ hence $x=N^{e-1} v$ and $L \subset N^{e-1} V$. If $c_{e}^{N} \in \mathbf{Z}^{\prime \prime}$ then we have $L \subset N^{e-1} V$ by (b). This proves (c).

We show:

(d) $N L \subset L, \quad N\left(L^{\perp}\right) \subset L^{\perp}$.

From (c) we see that $N L=0$ (since $N^{e}=0$ and $N R=0$ ). Thus the first inclusion holds. The second inclusion follows from the first.

We define a subset $\mathcal{M}_{Q}$ of $\tilde{\mathcal{M}}_{Q}$ as follows:

$\mathcal{M}_{Q}=\tilde{\mathcal{M}}_{Q}$ if $V \in \mathcal{C}^{\prime}$

$\mathcal{M}_{Q}=\left\{\tilde{N} \in \tilde{\mathcal{M}}_{Q} ; \operatorname{ker} \tilde{N} \in \mathcal{C}^{\prime \prime}\right\}$ if $V \in \mathcal{C}^{\prime \prime}$.

We show:

(e) If $N \in \mathcal{M}_{Q}-\{0\}$, then $\left.Q\right|_{L}=0$.

If $R \neq 0$ the result is obvious. Now assume that $R=0$. If $e \geq 3$ we have $\left.Q\right|_{N^{e-1} V}=0$ as in the proof (c). The same argument shows that $\left.Q\right|_{N^{e-1} V}=0$ if $\lambda=0$. If $\lambda=0$ or $e \geq 3$ we have $L \subset N^{e-1} V$, see (c), hence $\left.Q\right|_{L}=0$. Thus we may assume that $\lambda \neq 0, e=2$. Since $\operatorname{ker} N \in \mathcal{C}^{\prime \prime}$ and $V \in \mathcal{C}^{\prime \prime}$ we have $N V \in \mathcal{C}^{\prime \prime}$ that is $N^{e-1} V \in \mathcal{C}^{\prime \prime}$. As in the proof of (b) we see that $L$ is spanned by $N^{e-1} v(v$ as in that proof) which is contained in $\operatorname{ker}\left(Q:(\operatorname{ker} \lambda)^{\perp} \rightarrow \mathbf{k}\right)$; the result follows.

2.3. Let $N \in \mathcal{M}_{Q}-\{0\}, e=e_{N}, \lambda=\lambda_{e}^{N}, L=L_{N}$. We have $e \geq 2$. We set $V^{\prime}=L^{\perp} / L$, see 2.2(a). From 2.2(d) we see that $N$ induces a (nilpotent) endomorphism of $V^{\prime}$, denoted by $N^{\prime}$. Let $e^{\prime}=e_{N^{\prime}}$. If $\lambda=0$ we have $e^{\prime} \leq e-1$; if $\lambda \neq 0$ we have $e^{\prime} \leq e$. Define a quadratic form $Q^{\prime}: V^{\prime} \rightarrow \mathbf{k}$ by $Q^{\prime}\left(x^{\prime}\right)=Q(x)$ where $x$ is a representative of $x^{\prime} \in V^{\prime}$ in $L^{\perp}$. (To see that $Q^{\prime}$ is well defined we use 2.2(e).) The symplectic form associated to $Q^{\prime}$ is $\left\langle x^{\prime}, y^{\prime}\right\rangle^{\prime}=\langle x, y\rangle$ where $x^{\prime}, y^{\prime} \in V^{\prime}$ and $x, y$ are representatives of $x^{\prime}, y^{\prime}$ in $L^{\perp}$. Its radical is

$\left\{x \in L^{\perp} ;\left\langle x, L^{\perp}\right\rangle=0\right\} / L=(L+R) / L$.

It follows that $Q^{\prime}$ is nondegenerate. We have $N^{\prime} \in \tilde{\mathcal{M}}_{Q^{\prime}}$. We show:

(a) $N^{\prime} \in \mathcal{M}_{Q^{\prime}}$.

To do this we may assume that $V \in \mathcal{C}^{\prime \prime}$. Then $R=0$. It is enough to show that $\operatorname{dim} \operatorname{ker} N^{\prime}=\operatorname{dim} \operatorname{ker} N \bmod 2$. We have $\operatorname{ker} N^{\prime}=\left\{x \in L^{\perp} ; N x \in L\right\} / L$. We have an exact sequence

$$
0 \rightarrow L \stackrel{a}{\rightarrow} \operatorname{ker} N \stackrel{b}{\rightarrow}\left\{x \in L^{\perp} ; N x \in L\right\} / L \stackrel{c}{\rightarrow} L \cap N\left(L^{\perp}\right) \rightarrow 0
$$

where $a$ is the inclusion, $b$ is induced by the inclusion ker $N \subset L^{\perp}$ and $c$ is induced by $x \mapsto N x$. Thus $\operatorname{dim} L-\operatorname{dim} \operatorname{ker} N+\operatorname{dim} \operatorname{ker} N^{\prime}-\operatorname{dim}\left(L \cap N\left(L^{\perp}\right)\right)=0$ and it is enough to show that $\operatorname{dim} L=\operatorname{dim}\left(L \cap N\left(L^{\perp}\right)\right) \bmod 2$.

Assume first that $\lambda \neq 0$. We show that $L \subset N\left(L^{\perp}\right)$ (this implies that $\operatorname{dim} L=$ $\operatorname{dim}\left(L \cap N\left(L^{\perp}\right)\right)$ ). If $e=2$ this follows from 2.2(b). (We have $N V \in \mathcal{C}^{\prime \prime}$ since $\operatorname{ker} N \in \mathcal{C}^{\prime \prime}$ and $V \in \mathcal{C}^{\prime \prime}$.) Assume now that $e \geq 3$. Since $R=0$ we see from $2.2(\mathrm{c})$ that $L$ is spanned by $N^{e-1} x$ for some $x \in V$. It is enough to show that 
$N^{e-2} x \in L^{\perp}$; since $e \geq 3$ it is enough to show that $N V \subset L^{\perp}$; this is clear since $L^{\perp}$ is $N$-stable of codimension 1 .

Assume next that $\lambda=0$. We must show that

$\operatorname{dim} N^{e-1} V=\operatorname{dim}\left(N^{e-1} V \cap N\left(\operatorname{ker} N^{e-1}\right)\right) \bmod 2$.

If $e \geq 3$ we have $N^{e-1} V \subset N\left(\operatorname{ker} N^{e-1}\right)$ hence $\operatorname{dim} N^{e-1} V=\operatorname{dim}\left(N^{e-1} V \cap\right.$ $\left.N\left(\operatorname{ker} N^{e-1}\right)\right)$. If $e=2$ we have $N\left(\operatorname{ker} N^{e-1}\right)=0$ and it is enough to show that $N V \in \mathcal{C}^{\prime \prime}$. But this follows from $\operatorname{ker} N \in \mathcal{C}^{\prime \prime}$ and $V \in \mathcal{C}^{\prime \prime}$. This completes the proof of (a).

2.4. Let $N \in \mathcal{M}_{Q}-\{0\}$. Let $e, \lambda, L, V^{\prime}, N^{\prime}, Q^{\prime}$ be as in 2.2. Properties (i)-(iii) below describe the invariants $c_{i}^{N^{\prime}}, \epsilon_{i}^{N^{\prime}}$ of $N^{\prime}$.

(i) Assume that $\lambda=0$. We have $c_{e}^{N^{\prime}}=0 ; c_{e-2}^{N^{\prime}}=c_{e-2}^{N}+c_{e}^{N}$ (if $\left.e>2\right) ; c_{i}^{N^{\prime}}=c_{i}^{N}$ if $i \neq e, i \neq e-2 ; \epsilon_{i}^{N^{\prime}}=\epsilon_{i}^{N}$ for any $i$.

(ii) Assume that $\lambda \neq 0$ and $c_{e}^{N} \in \mathbf{Z}^{\prime \prime}$. Then $c_{e}^{N^{\prime}}=c_{e}^{N}-2 ; c_{e-1}^{N^{\prime}}=c_{e-1}^{N}+2$; $c_{i}^{N^{\prime}}=c_{i}^{N}$ for $i \neq e, i \neq e-1 ; \epsilon_{e}^{N^{\prime}}=0 ; \epsilon_{i}^{N^{\prime}}=\epsilon_{i}^{N}$ for any $i \neq e$.

(iii) Assume that $\lambda \neq 0, c_{e}^{N} \in \mathbf{Z}^{\prime}$. Then $c_{e}^{N^{\prime}}=c_{e}^{N}-1 ; c_{e-2}^{N^{\prime}}=c_{e-2}^{N}+1$ (if $e>2$ ); $c_{i}^{N^{\prime}}=c_{i}^{N}$ for $i \neq e, i \neq e-2 ; \epsilon_{e}^{N^{\prime}}=0 ; \epsilon_{e-2}^{N^{\prime}}=1$ (if $e>2$ ); $\epsilon_{i}^{N^{\prime}}=\epsilon_{i}^{N}$ for any $i \notin\{e, e-2\}$.

We have $N^{\prime e-1} V^{\prime}=\left(N^{e-1}\left(L^{\perp}\right)+L\right) / L=N^{e-1}\left(L^{\perp}\right) /\left(N^{e-1}\left(L^{\perp}\right) \cap L\right)$.

If $\lambda=0$ we have $L^{\perp}=\operatorname{ker} N^{e-1}$ and $N^{e-1}\left(L^{\perp}\right)=0$. Hence $N^{\prime e-1} V^{\prime}=0$ and $c_{i}^{N^{\prime}}=0$ for $i \geq e$.

If $\lambda \neq 0$ and $N^{e-1} V \in \mathcal{C}^{\prime \prime}$ (that is $c_{e}^{N} \in \mathbf{Z}^{\prime \prime}$ ) we have $L \subset N^{e-1}\left(L^{\perp}\right)$ (see 2.2(b)) so that $N^{\prime e-1} V^{\prime}=N^{e-1}\left(L^{\perp}\right) / L$. If $\lambda \neq 0$ and $N^{e-1} V \in \mathcal{C}^{\prime}$ (that is $c_{e}^{N} \in \mathbf{Z}^{\prime}$ ) we have $L \not \subset N^{e-1}\left(L^{\perp}\right)$ (see $2.2(\mathrm{~b})$ ) and, since $L$ is a line, we have $N^{e-1}\left(L^{\perp}\right) \cap L=0$ and $N^{\prime e-1} V^{\prime}=N^{e-1}\left(L^{\perp}\right)$. We have an exact sequence

$$
0 \rightarrow \operatorname{ker} N^{e-1} \cap L^{\perp} \rightarrow L^{\perp} \stackrel{N^{e-1}}{\longrightarrow} N^{e-1}\left(L^{\perp}\right) \rightarrow 0 .
$$

Now ker $N^{e-1} \subset$ ker $\lambda=L^{\perp}$ hence this exact sequence becomes

$$
0 \rightarrow \operatorname{ker} N^{e-1} \rightarrow \operatorname{ker} \lambda \rightarrow N^{e-1}\left(L^{\perp}\right) \rightarrow 0 \text {. }
$$

We see that

$\operatorname{dim} N^{e-1}\left(L^{\perp}\right)=\operatorname{dim} \operatorname{ker} \lambda-\operatorname{dim} \operatorname{ker} N^{e-1}=\operatorname{dim} V-\operatorname{dim} \operatorname{ker} N^{e-1}-1=c_{e}^{N}-1$ so that $c_{e}^{N^{\prime}}=\operatorname{dim} N^{\prime e-1} V^{\prime}$ equals $c_{e}^{N}-1$ if $c_{e}^{N} \in \mathbf{Z}^{\prime}$ and equals $c_{e}^{N}-2$ if $c_{e}^{N} \in \mathbf{Z}^{\prime \prime}$.

We write $V=W \oplus Y$ as in $1.9(\mathrm{c})$. Let $N_{1}=\left.N\right|_{W}, N_{2}=\left.N\right|_{Y}$. Let us now assume that we are not in the case

(*) $V \in \mathcal{C}^{\prime}, \lambda \neq 0, e=2, c_{e}^{N} \in \mathbf{Z}^{\prime}$.

By 2.2(c) we have $L \subset N^{e-1} V$. Since $N^{e-1} Y=0$ we have $L \subset N^{e-1} W$ hence $L \subset W$. Since $\operatorname{ker} N^{e-1} \subset \operatorname{ker} \lambda=L^{\perp}$ and $Y \subset \operatorname{ker} N^{e-1}$ we have $Y \subset L^{\perp}$. Hence we have canonically $V^{\prime}=W^{\prime} \oplus Y$ where $W^{\prime}=L^{\perp^{\prime}} / L^{\prime}$ (and $L^{\perp^{\prime}}=\{x \in$ $W ;\langle x, L\rangle=0\}$ ) with both $W^{\prime}, Y, N^{\prime}$-stable. Let $N_{1}^{\prime}=\left.N^{\prime}\right|_{W^{\prime}}$. If $\lambda=0$ then $c_{e-2}^{N_{1}^{\prime}}=c_{e}^{N_{1}}=c_{e}^{N}$ and $c_{i}^{N_{1}^{\prime}}=0$ for $i \neq e-2$. If $\lambda \neq 0$ we have either

$$
c_{e}^{N_{1}^{\prime}}=c_{e}^{N_{1}}-1=c_{e}^{N}-1, c_{e-2}^{N_{1}^{\prime}}=1, c_{i}^{N_{1}^{\prime}}=0 \text { for } i \notin\{e, e-2\}
$$

or 


$$
c_{e}^{N_{1}^{\prime}}=c_{e}^{N_{1}}-2=c_{e}^{N}-2, c_{e-1}^{N_{1}^{\prime}}=2, c_{i}^{N_{1}^{\prime}}=0 \text { for } i \notin\{e, e-1\} ;
$$

the formulas for $c_{i}^{N^{\prime}}$ in (i)-(iii) follow since

$$
c_{i}^{N^{\prime}}=c_{i}^{N_{1}^{\prime}}+c_{i}^{N_{2}}=c_{i}^{N_{1}^{\prime}}+c_{i}^{N} \text { for } i \leq e-1 .
$$

(Those formulas hold also in the excluded case $(*)$. Indeed in that case we have $c_{2}^{N^{\prime}}=c_{2}^{N}-1$ as we have seen already. For $i>2$ we have $c_{i}^{N^{\prime}}=0$ and $c_{1}^{N^{\prime}}+2 c_{2}^{N^{\prime}}=$ $\operatorname{dim} V^{\prime}=\operatorname{dim} V-2=c_{1}^{N}+2 c_{2}^{N}-2$ hence $c_{1}^{N^{\prime}}=c_{1}^{N}$.)

Assume now that $l \neq 0$. We show that $\epsilon_{e}^{N^{\prime}}=0$. It is enough to show that if $x \in L^{\perp}$ then $\left\langle x, N^{e-1} x\right\rangle=0$. This follows from $L^{\perp}=\operatorname{ker} \lambda$. We have $\epsilon_{e-1}^{N^{\prime}}=0$ since $e-1 \in \mathbf{Z}^{\prime}$. (This determines completely $\epsilon_{i}^{N^{\prime}}$ in the case where $e=2$.)

Assume that $\lambda \neq 0, c_{e}^{N} \in \mathbf{Z}^{\prime}, e>2$. We show that $\epsilon_{e-2}^{N^{\prime}}=1$. It is enough to show that there exists $x \in V$ such that $N^{e-2} x \in L$ and $\left\langle x, N^{e-3} x\right\rangle \neq 0$. (For such $x$ we have automatically $x \in L^{\perp}$ since $N^{e-1} x=0$ and $\operatorname{ker} N^{e-1} \subset \operatorname{ker} \lambda=L^{\perp}$.) By 2.2(c) we can find $y \in V$ such that $N^{e-1} y$ is a basis element of $L$. We have $\left\langle y, N^{e-1} y\right\rangle \neq 0$. (If not we would have $y \in L^{\perp}$ and $L \subset N^{e-1}\left(L^{\perp}\right)$ contradicting 2.2(b).) Let $x=N y$. We have $N^{e-2} x=N^{e-1} y \in L$ and $\left\langle x, N^{e-3} x\right\rangle=$ $\left\langle N y, N^{e-2} y\right\rangle=\left\langle y, N^{e-1} y\right\rangle \neq 0$, as required.

We may now assume that we are not in the case $(*)$. We use the decompositions $V=W \oplus Y, V^{\prime}=W^{\prime} \oplus Y$ as above. The proof of the remaining assertions on $\epsilon_{i}^{N^{\prime}}$ in (i)-(iii) is standard.

2.5. Let $N \in \mathcal{M}_{Q}$. We associate to $N$ a collection of subspaces $\left(V^{\geq a}\right)_{a \in \mathbf{Z}}=$ $\left(V_{N}^{\geq a}\right)_{a \in \mathbf{Z}}$ of $V$ using induction on $D$. Let $e=e_{N}$. If $N=0$ we set $V \geq a=V$ for $a \leq 0, V^{\geq a}=0$ for $a \geq 1$. Thus $V^{\geq a}$ are defined when $D \leq 1$. We may assume that $D \geq 2, N \neq 0$ and that $V^{\geq a}$ are already defined when $V$ is replaced by a vector space of dimension $<D$. We have $e \geq 2$. Let $\lambda, L, V^{\prime}, N^{\prime}, Q^{\prime}$ be as in 2.2, 2.3. Let $\rho: L^{\perp} \rightarrow V^{\prime}$ be the obvious map. Since $L \neq 0$, we have $\operatorname{dim} V^{\prime}<D$. By the induction hypothesis, $V^{\prime \geq a}=V_{N^{\prime}}^{\prime} \geq a$ is defined for any $a \in \mathbf{Z}$. If $\lambda=0$ we set $V^{\geq a}=V$ for $a \leq 1-e ; V^{\geq a}=\rho^{-1}\left(V^{\prime \geq a}\right)$ for $a \in[2-e, e-1] ; V^{\geq a}=0$ for $a \geq e$. If $\lambda \neq 0$ we set

$$
V^{\geq a}=V \text { for } a \leq-e ; V^{\geq a}=\rho^{-1}\left(V^{\prime \geq a}\right) \text { for } a \in[1-e, e] ; V^{\geq a}=0 \text { for } a \geq 1+e \text {. }
$$

This completes the definition of the subspaces $V \geq a$.

From the definition it is clear that $V^{\geq a+1} \subset V^{\geq a}$ for any $a$. Thus $\left(V^{\geq a}\right)$ is a filtration of $V$. We show:

(a) If $a \geq 1$ we have $\left.Q\right|_{V \geq a}=0$ and $V^{\geq 1-a}=\left(V^{\geq a}\right)^{\perp}$.

We use induction on $D$. For $N=0$ the result is obvious. Thus the result holds when $D \leq 1$. Now assume that $D \geq 2, N \neq 0$. Let $\lambda, L, V^{\prime}, Q^{\prime}, N^{\prime}$ be as above. By the induction hypothesis we have $\left.Q^{\prime}\right|_{V^{\prime} \geq a}=0$ and $V_{N^{\prime}}^{\prime \geq 1-a}=\left(V_{N^{\prime}}^{\prime \geq a}\right)^{\perp^{\prime}}$ with ${ }^{\perp^{\prime}}$ relative to $V^{\prime}$. It follows that $\left.Q\right|_{V \geq a}=0$ and $V^{\geq 1-a}=\left(V^{\geq a}\right)^{\perp}$ for $a \in[1, e-1]$ (if $\lambda=0$ ) and for $a \in[1, e]$ (if $\lambda \neq 0$ ). For $a \geq e$ (with $\lambda=0$ ) and for $a \geq 1+e$ (with $\lambda \neq 0$ ) the result is again true since $\left.Q\right|_{\{0\}}=0$ and $\{0\}^{\perp}=V$. This proves (a). 
2.6. Let $N \in \mathcal{M}_{Q}-\{0\}$. Let $\lambda, L, V^{\prime}, N^{\prime}, Q^{\prime}, e^{\prime}$ be as in $2.2,2.3$. Let $\rho: L^{\perp} \rightarrow V^{\prime}$ be the obvious map. Define $\perp^{\prime}$ in terms of $V^{\prime}$ in the same way as $\perp$ is defined in terms of $V$. If $e^{\prime} \geq 1$ we set $\lambda^{\prime}=\lambda_{e^{\prime}}^{N^{\prime}}$. If $e^{\prime} \geq 2$ we define $L^{\prime}, V^{\prime \prime}, N^{\prime \prime}, Q^{\prime \prime}, e^{\prime \prime}$ in terms of $V^{\prime}, N^{\prime}, Q^{\prime}$ in the same way as $L, V^{\prime}, N^{\prime}, Q^{\prime}, e^{\prime}$ were defined in terms of $V, N, Q$. If $e^{\prime} \geq 2$ and $e^{\prime \prime} \geq 1$ we set $\lambda^{\prime \prime}=\lambda_{e^{\prime \prime}}^{N^{\prime \prime}}$.

Let $V=W \oplus Y$ be as in $1.9(\mathrm{c})$. For a subspace $Z$ of $Y$ let $Z^{\vdash}=\{y \in Y ;\langle y, Z\rangle=$ $0\}$.

In the case where $\lambda=0, \epsilon^{\prime}=\epsilon-1 \geq 2, \lambda^{\prime} \neq 0$ we can view $L^{\prime}$ as a line in $Y$ as follows. We have $N^{e-1} V \subset W, Y \subset \operatorname{ker} N^{e-1}$ hence $V^{\prime}=W^{\prime} \oplus Y$ where $W^{\prime}=\left(\operatorname{ker} N^{e-1} \cap W\right) / N^{e-1} V$. Since $W^{\prime} \subset \operatorname{ker} N^{\prime e-2} \subset \operatorname{ker} \lambda^{\prime}$ we have $\left(\operatorname{ker} \lambda^{\prime}\right)^{\perp^{\prime}} \subset W^{\prime \perp^{\prime}}=Y$. Hence $L^{\prime} \subset Y$ and $L^{\prime \perp^{\prime}}=W^{\prime} \oplus L^{\prime \vdash}$.

In this subsection we describe explicitly $V^{\geq a}$ for certain $a$.

(i) If $\lambda \neq 0$ then $V^{\geq e}=L$.

We have $e^{\prime} \leq e$. If $e^{\prime}<e$ then $V^{\prime \geq e}=0$. If $e^{\prime}=e$ then (using 2.4(ii),(iii)) we have $\epsilon_{e}^{N^{\prime}}=0$ hence $\lambda^{\prime}=0$ and $V^{\prime \geq e}=0$. Hence $V^{\geq e}=\rho^{-1}(0)=L$.

(ii) If $\lambda \neq 0, e^{\prime}<e$ then $V^{\geq e-1}=L$.

If $e^{\prime}<e-1$ then $V^{\prime \geq e-1}=0$. If $e^{\prime}=e-1$ and $\lambda^{\prime}=0$ then $V^{\prime \geq e-1}=0$. If $e^{\prime}=e-1$ and $\lambda^{\prime} \neq 0$ then $e^{\prime}, e$ are even, contradiction. Hence $V^{\geq e-1}=\rho^{-1}(0)=L$.

(iii) If $\lambda=0$ and either $e^{\prime} \leq e-2$ or $e^{\prime}=e-1, \lambda^{\prime}=0$ then $V^{\geq e-1}=N^{e-1} V$. If $e^{\prime} \leq e-2$ we have $V^{\prime \geq e-1}=0$. If $e^{\prime}=e-1, \lambda^{\prime}=0$ we have again $V^{\prime \geq e-1}=0$. Hence $V^{\geq e-1}=\rho^{-1}(0)=L=N^{e-1} V$.

(iv) If $\lambda \neq 0, e^{\prime}=e$ then $V^{\geq e-1}=N^{e-1} L^{\perp}+L \subset \operatorname{ker} N$.

Using 2.4(ii),(iii) we have $\lambda^{\prime}=0$. Hence $e^{\prime \prime}<e^{\prime}$. If either $e^{\prime \prime} \leq e^{\prime}-2$ or $e^{\prime \prime}=e^{\prime}-1, \lambda^{\prime \prime}=0$ then from (iii) applied to $V^{\prime}$ we get $V^{\prime \geq e-1}=N^{\prime e-1} V^{\prime}$ and the result follows. If $e^{\prime \prime}=e^{\prime}-1, \lambda^{\prime \prime} \neq 0$ then $e^{\prime \prime}, e$ are even, contradiction. We have $N V^{\geq e-1}=0$ since $N^{e}=0$ and $N L=0$.

(v) If $\lambda=0$ and either $e^{\prime} \leq e-3$ or $e^{\prime}=e-2 \geq 1, \lambda^{\prime}=0$ or $e=2, e^{\prime}=0$ then $V^{\geq e-2}=N^{e-1} V$.

If $e^{\prime} \leq e-3$ then $V^{\prime \geq e-2}=0$. If $e^{\prime}=e-2 \geq 1, \lambda^{\prime}=0$ then $V^{\prime \geq e-2}=0$. If $e=2, e^{\prime}=0$ then $V^{\prime}=0$ and $V^{\prime \geq e-2}=0$. Hence $V^{\geq e-2}=\rho^{-1}(0)=L=N^{e-1} V$.

(vi) If $\lambda=0, e^{\prime}=e-1, \lambda^{\prime} \neq 0$ then $V^{\geq e-1}=\left\{x \in V ; N^{e-1} x=0,\left\langle x, N^{e-2} x\right\rangle=\right.$ $0\}^{\perp} \cap q^{-1}(0)$,

$V^{\geq e-1}=N^{e-1} V \oplus L^{\prime} \subset \operatorname{ker} N$.

By (i) for $V^{\prime}$ we have $V^{\prime \geq e-1}=L^{\prime}=\left\{x \in V^{\prime} ;\left\langle x, N^{e-2} x\right\rangle=0\right\}^{\perp^{\prime}} \cap q^{\prime-1}(0)$. Hence $V^{\geq e-1}=\operatorname{ker} N^{e-1} \cap\left\{x \in \operatorname{ker} N^{e-1} ;\left\langle x, N^{e-2} x\right\rangle=0\right\}^{\perp} \cap q^{-1}(0)$.

In the last equality of (vi) we regard $N^{e-1} V$ as a subspace of $W$ and $L^{\prime}$ as a subspace of $Y$ (as earlier in this subsection).

(vii) If $\lambda=0, e^{\prime}=e-2 \geq 1, \lambda^{\prime} \neq 0$ we have $V^{\geq e-2}=\left\{x \in V ; N^{e-1} x=\right.$ $\left.0,\left\langle x, N^{e-3} x\right\rangle=0\right\}^{\perp} \subset \operatorname{ker} N$.

By (i) for $V^{\prime}$ we have $V^{\prime \geq e-2}=\left\{x^{\prime} \in V^{\prime} ;\left\langle x^{\prime}, N^{\prime e-3} x^{\prime}\right\rangle 0\right\}^{\perp^{\prime}} \cap Q^{\prime-1}(0)$. Hence

$V^{\geq e-2}=\operatorname{ker} N^{e-1} \cap\left\{x \in \operatorname{ker} N^{e-1} ;\left\langle x, N^{e-3} x\right\rangle=0\right\}^{\perp} \cap Q^{-1}(0)$.

We have $N V \subset\left\{x \in \operatorname{ker} N^{e-1} ;\left\langle x, N^{e-3} x\right\rangle=0\right\}$ since $\lambda=0$. Taking $\perp$ we obtain $\left\{x \in \operatorname{ker} N^{e-1} ;\left\langle x, N^{e-3} x\right\rangle=0\right\}^{\perp} \subset \operatorname{ker} N$. Since ker $N \subset \operatorname{ker} N^{e-1}$ we see that 
$V \geq e-2$ is as required.

(viii) If $\lambda=0, e^{\prime}=e-1, \lambda^{\prime} \neq 0, e^{\prime \prime} \leq e^{\prime}-1$, we have $V^{\geq e-2}=\left\{x \in V ; N^{e-1} x=\right.$ $\left.0,\left\langle x, N^{e-2} x\right\rangle=0\right\}^{\perp} \cap Q^{-1}(0) \subset \operatorname{ker} N$.

By (ii) for $V^{\prime}$ we have $V^{\prime \geq e-2}=\left\{x^{\prime} \in V^{\prime} ;\left\langle x^{\prime}, N^{\prime e-2} x^{\prime}\right\rangle 0\right\}^{\perp} \cap Q^{\prime-1}(0)$. Hence

$V^{\geq e-2}=\operatorname{ker} N^{e-1} \cap\left\{x \in \operatorname{ker} N^{e-1} ;\left\langle x, N^{e-2} x\right\rangle=0\right\}^{\perp} \cap Q^{-1}(0)$.

We have $N V \subset\left\{x \in \operatorname{ker} N^{e-1} ;\left\langle x, N^{e-2} x\right\rangle=0\right\}$. Taking $\perp$ we obtain $\{x \in$ $\left.\operatorname{ker} N^{e-1} ;\left\langle x, N^{e-2} x\right\rangle=0\right\}^{\perp} \subset \operatorname{ker} N$. Since $\operatorname{ker} N \subset \operatorname{ker} N^{e-1}$ we see that $V^{\geq e-2}$ is as required.

(ix) If $\lambda=0, e^{\prime}=e-1, \lambda^{\prime}=0$ and either $e^{\prime}=1$ or $e \geq 3, e^{\prime \prime} \leq e^{\prime}-2$ or $e \geq 3, e^{\prime \prime}=e^{\prime}-1, \lambda^{\prime \prime}=0$ then $V^{\geq e-2}=N^{e-2}\left(\operatorname{ker} N^{e-1}\right)+N^{e-1} V \subset \operatorname{ker} N$.

By (iii) for $V^{\prime}$ we have $V^{\prime \geq e-2}=N^{\prime e-2} V^{\prime}$. Hence $V^{\geq e-2}$ is as required.

(x) If $\lambda=0, e^{\prime}=e-1, \lambda^{\prime} \neq 0, e^{\prime \prime}=e^{\prime}$, then $V^{\geq e-2}=N^{e-1} V \oplus\left(N^{e-2} L^{\prime \vdash}+L^{\prime}\right) \subset$ ker $N$.

Here we regard $N^{e-1} V$ as a subspace of $W$ and $L^{\prime}$ as a subspace of $Y$ (as earlier in this subsection). Then $N^{e-2} L^{\prime \vdash}+L^{\prime}$ is a subspace of $Y$. By (iv) for $V^{\prime}$ we have $V^{\prime \geq e-2}=N^{\prime e-2} L^{\prime \perp^{\prime}}+L^{\prime}$. Since $N^{\prime e-2} W^{\prime}=0$ we have $V^{\prime \geq e-2}=$ $N^{\prime e-2} L^{\prime \vdash}+L^{\prime} \subset Y$ and $V^{\geq e-2}=N^{e-1} V \oplus\left(N^{e-2} L^{\prime \vdash}+L^{\prime}\right)$. By (iv) for $V^{\prime}$ we have $N\left(N^{e-2} L^{\prime \vdash}+L^{\prime}\right)=0$. Hence $N V^{\geq e-2}=0$.

(xi) If $\lambda=0, e^{\prime}=e-1, e \geq 3, \lambda^{\prime}=0, e^{\prime \prime}=e^{\prime}-1, \lambda^{\prime \prime} \neq 0$ then $V^{\geq e-2}=$ $N^{e-1} V \oplus\left(\left\{b \in Y ; N^{e-2} b=0,\left\langle b, N^{e-3} b\right\rangle=0\right\}^{\vdash} \cap q^{-1}(0)\right) \subset \operatorname{ker} N$.

By (vi) for $V^{\prime}$ we have $V^{\prime \geq e-2}=U^{\prime \perp^{\prime}} \cap Q^{\prime-1}(0)$ where $U^{\prime}=\left\{x \in V^{\prime} ; N^{\prime e-2} x=\right.$ $0,\left\langle x, N^{\prime e-3} x^{\prime}=0\right\}$. We write $V^{\prime}=W^{\prime} \oplus Y$ as in the proof of $(\mathrm{x})$. We write the condition that $x=a+b$ with $a \in W^{\prime}, b \in Y$ is in $U^{\prime}$ in terms of $a, b$. Note that $N^{\prime e-2} a=0$ and $\left\langle a, N^{\prime e-3} a\right\rangle=0$. (The last equality follows from $\tilde{a} \in W, N^{e-1} \tilde{a}=0 \Longrightarrow\left\langle\tilde{a}, N^{e-3} \tilde{a}\right\rangle=0$. Indeed we have $\tilde{a}=N c$ with $c \in W$ and $\left\langle\tilde{a}, N^{e-3} \tilde{a}\right\rangle=\left\langle N c, N^{e-3} N c\right\rangle=\left\langle c, N^{e-1} c\right\rangle=0$ since $\lambda=0$.) We see that $U^{\prime}=W^{\prime} \oplus\left\{b \in Y ; N^{e-2} b=0,\left\langle b, N^{e-3} b\right\rangle=0\right\}$ and

$V^{\prime \geq e-2}=\left\{b \in Y ; N^{e-2} b=0,\left\langle b, N^{e-3} b\right\rangle=0\right\}^{\vdash} \cap Q^{\prime-1}(0)$,

$V^{\geq e-2}=N^{e-1} V \oplus\left(\left\{b \in Y ; N^{e-2} b=0,\left\langle b, N^{e-3} b\right\rangle=0\right\}^{\vdash} \cap Q^{-1}(0)\right)$.

By (vi) for $V^{\prime}$ we have $N\left(\left\{b \in Y ; N^{e-2} b=0,\left\langle b, N^{e-3} b\right\rangle=0\right\}^{\vdash} \cap Q^{-1}(0)\right)=0$. Hence $N V^{\geq e-2}=0$.

2.7. Let $N \in \mathcal{M}_{Q}, e=e_{N}$. Let $V^{\geq a}$ be as in 2.5. We show:

(a) $N V^{\geq a} \subset V^{\geq a+2}$ for any $a \in \mathbf{Z}$.

When $N=0$ the result is obvious. Now assume that $N \neq 0$. Then $e \geq 2$. Let $\lambda, L, V^{\prime}, N^{\prime}, Q^{\prime}$ be as in 2.2, 2.3. We may assume that (a) holds when $V, N$ are replaced by $V^{\prime}, N^{\prime}$. We may assume that (a) holds when $V, N$ are replaced by $V^{\prime}, N^{\prime}$.

Assume first that $\lambda=0$. If $a \geq e$ then $V^{\geq a}=0$ and (a) is obvious. If $a \in\{e-2, e-1\}$ then $N V^{\geq a}=0$ by 2.6 and (a) holds. Assume now that $a=-e$ or that $a=1-e, e \geq 3$. To prove (a) in this case it is enough to show that $N V \subset V^{\geq a+2}$ that is (using 2.5(a)) $N V \subset\left(V^{\geq-1-a}\right)^{\perp}$ or that $N^{\dagger}\left(V^{\geq-1-a}\right) \in R$. This follows from $N\left(V^{\geq-1-a}\right)=0$ which has been noted earlier. If $a=-1, e=2$ we have $N V=V^{\geq a+2}$ and (a) holds. If $a \leq-1-e$ then $V^{\geq a+2}=V$ and (a) 
is obvious. If $2-e \leq a \leq e-3$ then $V^{\geq a}=\rho^{-1}\left(V^{\prime \geq a}\right), V^{\geq a+2}=\rho^{-1}\left(V^{\prime \geq a+2}\right)$ (notation of 2.5). Since $N^{\prime} V^{\prime \geq a} \subset V^{\prime \geq a+2}$ we see that (a) holds.

Assume next that $\lambda \neq 0$. If $a \geq e+1$ then $V^{\geq a}=0$ and (a) is obvious. If $a \in\{e-1, e\}$ then $N V^{\geq a}=0$ by 2.6 and (a) holds. Assume now that $a \in$ $\{-e,-1-e\}$. To prove (a) in this case it is enough to show that $N V \subset V^{\geq a+2}$ that is (using 2.5(a)) $N V \subset\left(V^{\geq-1-a}\right)^{\perp}$ or that $N^{\dagger}\left(V^{\geq-1-a}\right) \in R$. This follows from $N\left(V^{\geq-1-a}\right)=0$ which has been noted earlier. If $a \leq-2-e$ then $V^{\geq a+2}=V$ and (a) is obvious. If $1-e \leq a \leq e-2$ then $V^{\geq a}=\rho^{-1}\left(V^{\prime \geq a}\right), V^{\geq a+2}=\rho^{-1}\left(V^{\prime \geq a+2}\right)$ (notation of 2.5). Since $N^{\prime} V^{\prime \geq a} \subset V^{\prime \geq a+2}$ we see that (a) holds. This proves (a).

For any $a \in \mathbf{Z}$ we set $\bar{V}^{a}=V^{\geq a} / V^{\geq a+1}$. From (a) we see that $N$ induces a linear map $\bar{N}: \bar{V}^{a} \rightarrow \bar{V}^{a+2}$.

2.8. In the setup of 2.6 taking $\perp$ in $2.6(\mathrm{i}),(\mathrm{iii}),(\mathrm{vi})$ we obtain If $\lambda \neq 0$ then $V^{\geq 1-e}=L^{\perp}$.

If $\lambda=0$ and either $e^{\prime} \leq e-2$ or $e^{\prime}=e-1, \lambda^{\prime}=0$ then $V^{\geq 2-e}=\operatorname{ker} N^{e-1}$.

If $\lambda=0, e^{\prime}=e-1, \lambda^{\prime} \neq 0$ then $V^{\geq 2-e}=\left(\operatorname{ker} N^{e-1} \cap W\right) \oplus L^{\prime \vdash}$.

2.9. For any $a \geq 0$ we set $K_{a}=\operatorname{ker} \bar{N}^{a}: \bar{V}^{-a} \rightarrow \bar{V}^{a}$. For any $a \in \mathbf{Z}_{\geq 2}^{\prime \prime}$ we define a quadratic form $Q_{a}: \bar{V}^{-a} \rightarrow \mathbf{k}$ by $Q_{a}(x)=Q_{0}\left(\bar{N}^{a / 2} x\right)$. (Note that $\bar{N}^{a / 2} x \in \bar{V}^{0}$.) If $\dot{x}$ is a representative of $x$ in $V^{\geq-a}$ we have

$$
Q_{a}(x)=Q\left(N^{a / 2} \dot{x}\right)=\left\langle N^{a / 2-1} \dot{x}, N^{a / 2} \dot{x}\right\rangle=\left\langle\dot{x}, N^{a-1} \dot{x}+c N^{a} \dot{x}+\ldots\right\rangle
$$

where $c \in \mathbf{k}$. If $f>0$ we have $\left\langle x, N^{a+f} x\right\rangle=0$ since $\left\langle V^{\geq-a}, V^{\geq a+2 f}\right\rangle=0$. Moreover,

$$
\begin{aligned}
& \left\langle x, N^{a} x\right\rangle=\left\langle N^{a / 2} x+c^{\prime} N^{a / 2+1} x+\ldots, N^{a / 2} x\right\rangle \\
& =\left\langle c^{\prime} N^{a / 2+1} x+\ldots, N^{a / 2} x\right\rangle=c^{\prime}\left\langle x, N^{a+1} x\right\rangle=0
\end{aligned}
$$

where $c^{\prime} \in \mathbf{k}$. Hence

$$
Q_{a}(x)=\left\langle\dot{x}, N^{a-1} \dot{x}\right\rangle .
$$

Let $\langle,\rangle_{a}$ be the symplectic form on $\bar{V}^{-a}$ associated to $Q_{a}$. Thus

$$
\left\langle x, x^{\prime}\right\rangle_{a}=\left\langle\bar{N}^{a / 2} x, \bar{N}^{a / 2} x^{\prime}\right\rangle_{0}
$$

for $x, x^{\prime} \in \bar{V}^{-a}$. If $\dot{x}, \dot{x}^{\prime}$ are representatives of $x, x^{\prime}$ in $V^{\geq-a}$ we have

$$
\left\langle x, x^{\prime}\right\rangle_{a}=\left\langle N^{a / 2} \dot{x}, N^{a / 2} \dot{x}^{\prime}\right\rangle=\left\langle\dot{x}, N^{a} \dot{x}^{\prime}+c N^{a+1} \dot{x}^{\prime}+\ldots\right\rangle=\left\langle\dot{x}, N^{a} \dot{x}^{\prime}\right\rangle=\left\langle x, \bar{N}^{a} x^{\prime}\right\rangle .
$$

(We use that $\left\langle V^{\geq-a}, V^{\geq a+2}\right\rangle=0$.) Let $R_{a}$ be the radical of $\langle,\rangle_{a}$. If $x^{\prime} \in R_{a}$ then $\left\langle x, \bar{N}^{a} x^{\prime}\right\rangle=0$ for all $x \in \bar{V}^{-a}$ hence $\bar{N}^{a} x^{\prime}=0$. Thus $R_{a}=K_{a}$. We show:

(a) If $a \in \mathbf{Z}^{\prime}$ we have $K_{a}=0$. If $a \in \mathbf{Z}_{\geq 0}^{\prime \prime} 0$ then $Q_{a}$ is nondegenerate; hence $\operatorname{dim} K_{a} \in\{0,1\}$.

If $N=0$ we have $V^{\geq-a}=V_{\geq 1-a}=V$ hence $\bar{V}^{-a}=0$ so that $K_{a}=0$ as required. Now assume that $N \neq 0$ so that $e \geq 2$. Let $\lambda, L, V^{\prime}, N^{\prime}, Q^{\prime}$ be as in $2.2,2.3$. Let $\bar{V}^{\prime a}, \bar{N}^{\prime a}, Q_{a}^{\prime}$ be the analogues of $\bar{V}^{a}, \bar{N}^{a}, Q_{a}$ for $V^{\prime}, N^{\prime}$ instead of $V, N$. We 
may assume that the analogue of (a) holds when $V, N$ is replaced by $V^{\prime}, N^{\prime}$. If $\lambda \neq 0, a>e$ or if $\lambda=0, a \geq e$ we have $\bar{V}^{-a}=0$ hence $K_{a}=0$ as required. If $\lambda \neq 0, a=e$ we have $\bar{V}^{-e}=V / L^{\perp}, \bar{V}^{e}=L, b N^{e}=0$ hence $K_{a}=V / L^{\perp}$. We must show that $Q_{a}$ is not identically zero. It is enough to show that $\dot{x} \mapsto\left\langle\dot{x}, N^{a-1} \dot{x}\right\rangle$ is not identically zero on $V$; this holds since $\lambda \neq 0$. If $\lambda=0, a=e-1, \epsilon_{e-1}^{N^{\prime}}=0$ then $\bar{V}^{1-e}=V / \operatorname{ker} N^{e-1}, \bar{V}^{e-1}=N^{e-1} V$ and $\bar{N}^{a}: \bar{V}^{-a} \rightarrow \bar{V}^{a}$ is an isomorphism; hence $K_{a}=0$, as required. If $\lambda=0, a=e-1, \epsilon_{e-1}^{N^{\prime}}=1$ then with notation of 2.6(vi), 2.8 we have $\bar{V}^{1-e}=W /\left(\operatorname{ker} N^{e-1} \mid W\right) \oplus Y / L^{\prime \vdash}, \bar{V}^{e-1}=N^{e-1} V \oplus L^{\prime}$; now $\bar{N}^{e-1}$ restricts to an isomorphism $W /\left(\operatorname{ker} N^{e-1} \mid W\right) \rightarrow N^{e-1} V$ and to the zero map $Y / L^{\prime \vdash} \rightarrow L^{\prime}$ (since $\left.N^{e-1} Y=0\right)$. Hence $K_{a}=Y / L^{\prime \vdash}$. We must show that $Q_{a}$ is not identically 0 on $Y / L^{\prime \vdash}$ or that $x \mapsto\left\langle x, N^{e-2} x\right\rangle$ is not identically 0 on $Y$. But this follows from $\epsilon_{e-1}^{N^{\prime}}=1$. If $\lambda \neq 0, a \in[1, e-1]$ or if $\lambda=0, a \in[1, e-2]$, we have $\bar{V}^{-a}=\bar{V}^{\prime-a}, \bar{V}^{a}=\bar{V}^{\prime a}$. We can identify $\bar{N}^{\prime a}=\bar{N}^{a}$ and (if $a \in \mathbf{Z}^{\prime \prime}$ ) $Q_{a}$ with $Q_{a}^{\prime}$. Hence the result follows from the induction hypothesis. If $a=0$ the result is obvious. This completes the proof.

We show:

(b) Assume that $a \in \mathbf{Z}_{\geq 2}^{\prime \prime}$. We set $\phi_{a}=\left|\left\{b \in \mathbf{Z}^{\prime \prime} ; c_{b}^{N} \in \mathbf{Z}^{\prime}, b>a\right\}\right|, \xi_{a}=\operatorname{dim} K_{a}$. We have $c_{a}^{N} \in \mathbf{Z}^{\prime} \Longrightarrow \xi_{a}=1 ; c_{a}^{N} \in \mathbf{Z}^{\prime \prime}, \phi_{a} \in \mathbf{Z}^{\prime} \Longrightarrow \xi_{a}=1 ; c_{a}^{N} \in \mathbf{Z}^{\prime \prime}, \phi_{a} \in$ $\mathbf{Z}^{\prime \prime} \Longrightarrow \xi_{a}=\epsilon_{a}^{N}$.

If $N=0$ the result is obvious. Now assume that $N \neq 0$ so that $e \geq 2$. Let $\lambda, V^{\prime}, N^{\prime}, Q^{\prime}$ be as in 2.2, 2.3. Let $\xi_{a}^{\prime}, \phi_{a}^{\prime}$ be the analogues of $\xi_{a}, \phi_{a}$ for $V^{\prime}, N^{\prime}$ instead of $V, N$. We may assume that the analogue of (b) holds when $V, N$ is replaced by $V^{\prime}, N^{\prime}$. If $\lambda \neq 0, a>e$ or if $\lambda=0, a \geq e$ we have (by the proof of (a)) $\xi_{a}=0$, as required. If $\lambda \neq 0, a=e$ we have (by the proof of (a)) $\xi_{a}=1$ as required. If $\lambda=0, a=e-1$ then (by the proof of (a)) $\xi_{a}=\epsilon_{e-1}^{N^{\prime}}=\epsilon_{e-1}^{N}$ as required. In the remainder of the proof we assume that either $\lambda \neq 0, a \in[1, e-1]$ or $\lambda=0, a \in[1, e-2]$. Then (by the proof of (a)), $\xi_{a}=\xi_{a}^{\prime}$. Using the induction hypothesis we see that if $c_{a}^{N^{\prime}} \in \mathbf{Z}^{\prime \prime}, \phi_{a}^{\prime} \in \mathbf{Z}^{\prime \prime}$ then $\xi_{a}=\epsilon_{a}^{\prime}{ }^{N}$; otherwise, $\xi_{a}=1$.

Assume first that $\lambda \neq 0$. Then $e \in \mathbf{Z}^{\prime \prime}$ hence $a \leq e-2$.

If $a=e-2, c_{e-2}^{N} \in \mathbf{Z}^{\prime}$ and $c_{e}^{N} \in \mathbf{Z}^{\prime}$ then $c_{e-2}^{N^{\prime}} \in \mathbf{Z}^{\prime \prime}, c_{e}^{N^{\prime}} \in \mathbf{Z}^{\prime \prime}$ and $\epsilon_{e-2}^{N^{\prime}}=1$ so that $\xi_{e-2}=1$.

If $a=e-2, c_{e-2}^{N} \in \mathbf{Z}^{\prime}$ and $c_{e}^{N} \in \mathbf{Z}^{\prime \prime}$ then $c_{e-2}^{N^{\prime}} \in \mathbf{Z}^{\prime}, c_{e}^{N^{\prime}} \in \mathbf{Z}^{\prime \prime}$ so that $\xi_{e-2}=1$. If $a=e-2, c_{e-2}^{N} \in \mathbf{Z}^{\prime \prime}$ and $c_{e}^{N} \in \mathbf{Z}^{\prime}$ then $c_{e-2}^{N^{\prime}} \in \mathbf{Z}^{\prime}, c_{e}^{N^{\prime}} \in \mathbf{Z}^{\prime \prime}$ and $\epsilon_{e-2}^{N^{\prime}}=1$ so that $\xi_{e-2}=1$. Also, $\phi_{e-2}=1$.

If $a=e-2, c_{e-2}^{N} \in \mathbf{Z}^{\prime \prime}$ and $c_{e}^{N} \in \mathbf{Z}^{\prime \prime}$ then $c_{e-2}^{N^{\prime}} \in \mathbf{Z}^{\prime \prime}, c_{e}^{N^{\prime}} \in \mathbf{Z}^{\prime \prime}, x_{e-2}=\epsilon_{e-2}^{N^{\prime}}=$ $\epsilon_{e-2}^{N}$.

If $a \leq e-4$ we have $c_{a}^{N^{\prime}}=c_{a}^{N}$. If these are odd then $\xi_{a}=1$. If these are even then $\phi_{a}=\phi_{a}^{\prime} \bmod 2\left(\right.$ if $c_{e}^{N} \in \mathbf{Z}^{\prime}, c_{e-2}^{N} \in \mathbf{Z}^{\prime}$ then $\phi_{a}=\phi_{a}^{\prime}+2$; otherwise, $\left.\phi_{a}=\phi_{a}^{\prime}\right)$.

Assume next that $\lambda=0$.

If $a=e-2$ then $c_{e}^{N} \in \mathbf{Z}^{\prime \prime}$ and $c_{a}^{N^{\prime}}=c_{a}^{N} \bmod 2$. If $c_{a}^{N^{\prime}}, c_{a}^{N}$ are odd we have $\xi_{a}=1$. If $c_{a}^{N^{\prime}}, c_{a}^{N}$ are even we have $\xi_{a}=\epsilon_{a}^{N^{\prime}}=\epsilon_{a}^{N}$.

If $a \leq e-3$ we have $c_{a}^{N^{\prime}}=c_{a}^{N}$. If these are both odd then $\xi_{a}=1$; if these are 
both even then $\phi_{a}=\phi_{a}^{\prime}$.

We see that $(b)$ holds.

We show:

(c) If $a \in Z Z_{\geq 1}^{\prime}$ then the bilinear pairing $\bar{V}^{-a} \times \bar{V}^{-a} \rightarrow \mathbf{k}, x, y \mapsto\left\langle x, \bar{N}^{a} y\right\rangle$ is symplectic; it is nondegenerate by (a). Hence $\bar{V}^{-a} \in \mathcal{C}^{\prime \prime}$.

If $N=0$ we have $\bar{V}^{-a}=0$ if $a \neq 0$ and (c) is obvious. Assume that $N \neq 0$. Then $e \geq 2$. Let $\lambda, V^{\prime}, N^{\prime}$ be as in 2.2, 2.3. We may assume that (c) holds when $V, N$ are replaced by $V^{\prime}, N^{\prime}$. If $\lambda \neq 0, a>e$ or if $\lambda=0, a \geq e$ we have $\bar{V}^{-a}=0$ and (c) is obvious. The case $\lambda \neq 0, a=e$ cannot occur since $e \in \mathbf{Z}^{\prime \prime}$. If $\lambda=0, a=e-1$ then $e-1 \in \mathbf{Z}^{\prime}$ and $\epsilon_{e-1}^{N^{\prime}}=0$; we have $\bar{V}^{1-e}=V / \operatorname{ker} N^{e-1}$ and $\left\langle x, N^{e-1} x\right\rangle=0$ for all $x$. Hence (c) holds. If $\lambda \neq 0, a \in[1, e-1]$ or if $\lambda=0, a \in[1, e-2]$, we have $\bar{V}^{-a}=\bar{V}^{\prime-a}$. Hence the result follows the induction hypothesis. The case $a=0$ does not arise. This completes the proof.

2.10. Let $X^{*}=\left(X^{\geq a}\right)_{a \in \mathbf{Z}}$ be a $Q$-filtration of $V$ (see 1.4). Let $N \in \mathcal{M}_{Q}, e=e_{N}$. We say that $X^{*}$ is $N$-adapted if conditions (i)-(iii) below hold.

(i) $N X^{\geq a} \subset X^{\geq a+2}$ for any $a$;

For any $a \geq 0$ let $\mathcal{K}_{a}$ be the kernel of the map $\nu^{a}: \operatorname{gr}^{-a} X^{*} \rightarrow \operatorname{gr}^{a} X^{*}$ induced by $N^{a}$. We state conditions (ii),(iii).

(ii) for any $a \in Z Z_{\geq 1}^{\prime}$ we have $\mathcal{K}_{a}=0$;

(iii) for any $a \in \mathbf{Z}_{\geq 2}^{\prime \prime}$ we have $\mathcal{K}_{a}=0$ or $\operatorname{dim} \mathcal{K}_{a}=1$; in the latter case the map $\mathcal{K}_{a} \rightarrow \mathbf{k}, x \mapsto\left\langle\dot{x}, N^{a-1} \dot{x}\right\rangle$ is a bijection.

(For $x \in \operatorname{gr}^{a} X^{*}$ we denote by $\dot{x}$ a representative of $x$ in $X^{\geq a}$.) Note that $\left(V^{\geq a}\right)_{a \in \mathbf{Z}}$ is $N$-adapted where $V^{\geq a}=V_{N}^{\geq a}$. We show:

(a) If $\left(X^{\geq a}\right)_{a \in \mathbf{Z}}$ is an $N$-adapted filtration of $V$ then $X^{\geq a}=V^{\geq a}$ for any $a$. If $V=0$ the result is obvious. Now assume that $V \neq 0$. If $N=0$ and $a \geq 1$ then $\nu^{a}: \operatorname{gr}^{-a} X^{*} \rightarrow \operatorname{gr}^{a} X^{*}$ is 0 and $\mathcal{K}_{a}=0$. Hence $X^{\geq a}=X^{\geq a+1}$ and $X^{\geq-a}=$ $X^{\geq-a+1}$ so that we have $X^{\geq 1}=X^{\geq 2}=\cdots=0$ and $X_{\geq 0}=X_{\geq-1}=\cdots=V$ as required. We now assume that $N \neq 0$ hence $e \geq 2$. Let $\lambda, L, V^{\prime}, N^{\prime}, Q^{\prime}, \rho, \epsilon^{\prime}$ be as in 2.2, 2.3. If $\epsilon^{\prime} \geq 1$ let $\lambda^{\prime}$ be as in 2.6. If $\epsilon^{\prime} \geq 2$ let $Y, L^{\prime}, L^{\prime \vdash}$ be as in 2.6. We may assume that (a) holds when $V, N$ are replaced by $V^{\prime}, N^{\prime}$.

If $a \geq e+1$ or if $a=e, \lambda=0$, then $\nu^{a}: \operatorname{gr}^{-a} X^{*} \rightarrow \operatorname{gr}^{a} X^{*}$ is 0 and $\mathcal{K}_{a}=0$. Hence $X^{\geq a}=X^{\geq a+1}$ and $X^{\geq-a}=X^{\geq-a+1}$ so that if $\lambda=0$ we have

$$
X^{\geq e}=X^{\geq e+1}=\cdots=0 \text { and } X_{\geq 1-e}=X_{\geq-e}=\cdots=V \text {; }
$$

if $\lambda \neq 0$ we have

$$
X^{\geq e+1}=X^{\geq e+2}=\cdots=0 \text { and } X_{\geq-e}=X_{\geq-1-e}=\cdots=V .
$$

Hence $X^{\geq a}=V^{\geq a}$ if $a \geq e+1$ or if $a=e, \lambda=0$ or if $a \leq-e$ or if $a=1-e, \lambda=0$.

Assume that $\lambda \neq 0$. Then $\mathcal{K}_{e}$ is the kernel of $0=\nu^{e}: V / X^{\geq 1-e} \rightarrow X^{\geq e}$ that is $\mathcal{K}_{e}=V / X^{\geq 1-e}$. Also $\mathcal{K}_{e} \rightarrow \mathbf{k}, x \mapsto\left\langle\dot{x}, N^{e-1} \dot{x}\right\rangle$ is not identically zero hence $\operatorname{dim} V / X^{\geq 1-e}=1$. Since for $x \in \operatorname{gr}^{1-e} X^{*}$ we have $\left.\dot{x}, N^{e-1} \dot{x}\right\rangle=0$ we see that $X^{\geq 1-e} \subset \operatorname{ker} \lambda=L^{\perp}$. Since $\operatorname{dim} X^{\geq 1-e}=\operatorname{dim} L^{\perp}$ we see that $X^{\geq 1-e}=L^{\perp}$. We have $X^{\geq e}=\left(X^{\geq 1-e}\right)^{\perp} \cap Q^{-1}(0)=L$ as required.

Next we assume that $\lambda=0$. Then $\mathcal{K}_{e-1}$, the kernel of $\nu^{a}: V / X^{\geq 2-e} \rightarrow X^{\geq e-1}$, 
is

$$
\begin{aligned}
& \left\{x \in V / X^{2-e} ; N^{e-1} \dot{x} \in X^{\geq e}=0\right\}=\left\{x \in V / X^{2-e} ; N^{e-1} \dot{x}=0\right\} \\
& =\operatorname{ker} N^{e-1} / X^{\geq 2-e} .
\end{aligned}
$$

If $\epsilon_{e-1}^{N}=0$ then $\left.\dot{x}, N^{e-2} \dot{x}\right\rangle=0$ for $x$ in this kernel that is for $x \in \mathcal{K}_{e-1}$. Hence in this case we have $\mathcal{K}_{e-1}=0$. Thus $N^{e-1}$ induces an isomorphism $V / X^{\geq 2-e} \rightarrow X^{\geq e-1}$ so that $X^{\geq e-1}=N^{e-1} V, X^{\geq 2-e}=\operatorname{ker} N^{e-1}$, as required. We have $X^{\geq 2-e}=\left(X^{\geq e-1}\right)^{\perp}=\left(V^{\geq e-1}\right)^{\perp}=V^{\geq 2-e}$ as required.

Now assume that $\lambda=0, \epsilon_{e-1}^{N}=1$. In this case we have an isomorphism ker $N^{e-1} / X^{2-e} \rightarrow \mathbf{k}$ induced by $\lambda_{e-1}^{N}$ that is we have

$$
X^{\geq 2-e}=\left\{x \in \operatorname{ker} N^{e-1} ; \lambda_{e-1}^{N} x=0\right\} .
$$

This is the same as $\left(\operatorname{ker} N^{e-1} \cap W\right) \oplus L^{\prime \vdash}=V^{\geq 2-e}$, see 2.8. Taking $\perp$ in $X^{\geq 2-e}=$ $V^{\geq 2-e}$ and intersecting with $Q^{-1}(0)$ we obtain $X^{\geq e-1}=V^{\geq e-1}$.

If $\lambda=0, a \in[2-e, e-1]$ we have $N^{e-1} V \subset X^{\geq a} \subset \operatorname{ker} N^{e-1}$ and we denote by $X^{\prime \geq a}$ the image of $X^{\geq a}$ under $\rho: \operatorname{ker} N^{e-1} \rightarrow V^{\prime}=\operatorname{ker} N^{e-1} / N^{e-1} V$. For $a \leq 1-e$ we set $X^{\prime \geq a}=V^{\prime}$ and for $a \geq e$ we set $X^{\prime \geq a}=0$. Now $\left(X^{\prime \geq a}\right)_{a \in \mathbf{Z}}$ is an $N^{\prime}$-adapted filtration of $V^{\prime}$. (We must only show the analogue of (ii),(iii) for $N^{\prime}$ with $a=e-1$. We have $X^{\prime \geq 1-e} / X^{\prime \geq 2-e}=\operatorname{ker} N^{e-1} / X^{\geq 2-e}$; this is $Y / L^{\prime \vdash}$ if $\epsilon^{\prime}=\epsilon-1, \lambda^{\prime} \neq 0$ and 0 otherwise. We have $X^{\prime \geq e-1} / X^{\prime \geq e}=X^{\geq e-1} / N^{e-1} V$. This is $L^{\prime}$ if $\epsilon^{\prime}=\epsilon-1, \lambda^{\prime} \neq 0$ and 0 otherwise. Hence (ii),(iii) are obvious in this case.) By the induction hypothesis we have $X^{\prime \geq a}=V^{\prime \geq a}$ for all $a$. Taking inverse image under $\rho$ we see that for $a \in[2-e, e-1]$ we have $X^{\geq a}=V^{\geq a}$.

If $\lambda \neq 0, a \in[1-e, e]$ we have $L \subset X^{\geq a} \subset L^{\perp}$ and we denote by $X^{\prime \geq a}$ the image of $X^{\geq a}$ under $\rho: L^{\perp} \rightarrow V^{\prime}=L^{\perp} / L$. For $a \leq-e$ we set $X^{\prime \geq a}=V^{\prime}$ and for $a \geq e+1$ we set $X^{\prime \geq a}=0$. Now $\left(X^{\prime \geq a}\right)_{a \in \mathbf{Z}}$ is an $N^{\prime}$-adapted filtration of $V^{\prime}$. (We have $X^{\prime \geq-e} / X^{\prime \geq 1-e}=0, X^{\prime \geq e} / X^{\prime \geq e+1}=0$.) By the induction hypothesis we have $X^{\prime \geq a}=V^{\prime \geq a}$ for all $a$. Taking inverse image under $\rho$ we see that for $a \in[1-e, e]$ we have $X^{\geq a}=V^{\geq a}$. This completes the proof of (a).

2.11. Assume that $V \in \mathcal{C}^{\prime \prime}$. If $g \in O_{Q}$ and $S \in \mathcal{J}_{Q}$, we set $\delta_{g}=\operatorname{dim}(S /(S \cap g(S)))$ mod 2. It is known that $\delta_{g}$ is independent of the choice of $S$ and that $\delta_{g}=0$ if and only if $g \in S O_{Q}$.

Let $N \in \tilde{\mathcal{M}}_{Q}$. We show that

(a) $\delta_{1+N}=\operatorname{dim} \operatorname{ker} N \bmod 2$.

If $N=0$ this is clear. Assume now that $N \neq 0$. Let $e=e_{N}, L=L_{N}$. We have $e \geq 2$. Let $\lambda, L$ be as in 2.2. We may assume that $V \in \mathcal{C}^{\prime \prime}$. Assume first that $e \geq 3$. As in the proof of $2.2(\mathrm{e})$ we have $\left.Q\right|_{L}=0$. We set $V^{\prime}=L^{\perp} / L$. The nondegenerate quadratic form $Q^{\prime}: V^{\prime} \rightarrow \mathbf{k}$ can be defined as in 2.3. The nilpotent endomorphism $N^{\prime}: V^{\prime} \rightarrow V^{\prime}$ induced by $N$ belongs to $\tilde{\mathcal{M}}_{Q^{\prime}}$. As in the proof of 2.3(a) we see that $\operatorname{dim} \operatorname{ker} N^{\prime}=\operatorname{dim} \operatorname{ker} N \bmod 2$. We have $\delta_{1+N}=\delta_{1+N^{\prime}}$. Since the result may be assumed to hold for $N^{\prime}$ we see that (a) holds. We now assume that $e=2$ that is $N^{2}=0, N \neq 0$. 
Assume that $\lambda \neq 0$. We can find $x \in V$ such that $\langle x, N x\rangle \neq 0$. Then $x, N x$ span a two-dimensional $N$-stable subspace $P$ of $V$ on which $\langle$,$\rangle is non-degenerate.$ Let $V^{\prime}=P^{\perp}$ and let $Q^{\prime}=\left.Q\right|_{V^{\prime}}$. Then $V=P \oplus V^{\prime}, Q^{\prime}$ is non-degenerate and $N$ restricts to a nilpotent map $N^{\prime}: V^{\prime} \rightarrow V^{\prime}$. Note that $\operatorname{dim} \operatorname{ker} N=\operatorname{dim} \operatorname{ker} N^{\prime}+1$ and $\delta_{1+N}=\delta_{1+N^{\prime}}+1 \bmod 2$. Since the result may be assumed to hold for $N^{\prime}$ we see that (a) holds.

Assume that $\lambda=0$. We write $V=W \oplus Y$ as in $1.9(\mathrm{c})$. Then $W, Y$ are $N$ stable nondegenerate even dimensional subspaces of $V$ with $\langle W, Y\rangle=0$; moreover $N Y=0$. Hence $\operatorname{dim} \operatorname{ker} N=\operatorname{dim} \operatorname{ker}\left(\left.N\right|_{W}\right)+\operatorname{dim} Y, \delta_{1+N}=\delta_{1+\left.N\right|_{W}}$. If $Y \neq 0$ we may assume that the result holds for $\left.N\right|_{W}$; we see that (a) holds. Thus we may assume that $Y=0$. We have $V=E \oplus N E$ with $E$ as in 1.9(a). Note that $\operatorname{dim} N E=D / 2$, see $1.9(\mathrm{~b})$. Since $\lambda=0$ we have $\left.Q\right|_{N E}=0$. Clearly $N E$ is $1+N$ stable. We see that $\delta_{1+N}=0$. The nondegenerate symmetric bilinear form on $E$ described in 1.9 (a) is symplectic since $\lambda=0$. Hence $\operatorname{dim} E \in \mathbf{Z}^{\prime \prime}$ and $\operatorname{dim} N E \in \mathbf{Z}^{\prime \prime}$. We see that $\operatorname{dim} \operatorname{ker} N \in \mathbf{Z}^{\prime \prime}$. Thus (a) holds.

From (a) we deduce that for $N \in \tilde{\mathcal{M}}_{Q}$ we have $N \in \mathcal{M}_{Q}$ if and only if $1+N \in$ $S O_{Q}$

2.12. We prove Theorem 1.7 (with $p=2$ ) in the form 1.7 (a). Let $T \in S O_{Q}$ be unipotent. Then $N=T-1 \in O_{Q}$ and by 2.11 , we have $N=T-1 \in \mathcal{M}_{Q}$. In 2.5 we have attached to $N$ a $Q$-filtration $X^{*}=\left(V_{N}^{\geq a}\right)$ of $V$. In 2.7, 2.9 we have shown that $N \in E_{*}^{\geq 2} X^{*}$. In 2.10 we have shown that the last property determines $X^{*}$ uniquely. Thus 1.7(a) is established.

\section{THE CASE $p \neq 2$}

3.1. In this section we assume that $p \neq 2$. In this case we have $R=0$.

Let $N \in \tilde{\mathcal{M}}_{Q}-\{0\}, e=e_{N}$. We have $e \geq 2$. Let $L=L_{N}=N^{e-1} V$, a subspace of $V$. By 1.1(b) we have $L^{\perp}=\operatorname{ker} N^{e-1}$. Since $2 e-2 \geq e$ we have $N^{e-1} V \subset \operatorname{ker} N^{e-1}$ hence:

(a) $L \subset L^{\perp}$.

Clearly,

(b) $N L=0, N V \subset L^{\perp}$.

We show:

(c) $\left.Q\right|_{L}=0$.

Let $v \in V$. We have $q\left(N^{e-1} v\right)=\left\langle N^{e-2} v, N^{e-1} v\right\rangle= \pm\left\langle v, N^{2 e-3} v\right\rangle$. If $e \geq 3$ this is 0 since $2 e-3 \geq e$. If $e=2$ this is 0 since $\langle v, N v\rangle=-\langle N v, v\rangle$ so that $2\langle v, N v\rangle=0$ and $\langle v, N v\rangle=0$.

3.2. Let $N \in \tilde{\mathcal{M}}_{Q}-\{0\}, e=e_{N}, L=L_{N}$. We have $e \geq 2$. We set $V^{\prime}=L^{\perp} / L$, see 3.1(a). From 3.1(b) we see that $N$ induces a (nilpotent) endomorphism of $V^{\prime}$, denoted by $N^{\prime}$. Let $e^{\prime}=e_{N^{\prime}}$. We have $e^{\prime} \leq e-1$. Define a quadratic form $Q^{\prime}: V^{\prime} \rightarrow \mathbf{k}$ by $Q^{\prime}\left(x^{\prime}\right)=Q(x)$ where $x$ is a representative of $x^{\prime} \in V^{\prime}$ in $L^{\perp}$. (To see that $Q^{\prime}$ is well defined we use 3.1(c).) The symmetric bilinear form associated to 
$Q^{\prime}$ is $\left\langle x^{\prime}, y^{\prime}\right\rangle^{\prime}=\langle x, y\rangle$ where $x^{\prime}, y^{\prime} \in V^{\prime}$ and $x, y$ are representatives of $x^{\prime}, y^{\prime}$ in $L^{\perp}$. Its radical is $\left\{x \in L^{\perp} ;\left\langle x, L^{\perp}\right\rangle=0\right\} / L=0$. It follows that $Q^{\prime}$ is nondegenerate. We have $N^{\prime} \in \tilde{\mathcal{M}}_{Q^{\prime}}$.

3.3. Let $N \in \tilde{\mathcal{M}}_{Q}, e=e_{N}$. We associate to $N$ a collection of subspaces

$$
\left(V^{\geq a}\right)_{a \in \mathbf{Z}}=\left(V_{N}^{\geq a}\right)_{a \in \mathbf{Z}}
$$

of $V$ using induction on $D$. If $N=0$ we set $V^{\geq a}=V$ for $a \leq 0, V^{\geq a}=0$ for $a \geq 1$. Thus $V^{\geq a}$ are defined when $D \leq 1$. We may assume that $D \geq 2, N \neq 0$ and that $V^{\geq a}$ are already defined when $V$ is replaced by a vector space of dimension $<D$. We have $e \geq 2$. Let $L, V^{\prime}, N^{\prime}, Q^{\prime}$ be as in 3.1, 3.2. Let $\rho: L^{\perp} \rightarrow V^{\prime}$ be the obvious map. Since $L \neq 0$, we have $\operatorname{dim} V^{\prime}<D$. By the induction hypothesis, $V^{\prime} \geq a=V_{N^{\prime}}^{\prime} \geq a$ is defined for any $a \in \mathbf{Z}$. We set

$V^{\geq a}=V$ for $a \leq 1-e ; V^{\geq a}=\rho^{-1}\left(V^{\prime \geq a}\right)$ for $a \in[2-e, e-1] ; V^{\geq a}=0$ for $a \geq e$.

This completes the definition of the subspaces $V^{\geq a}$.

From the definition it is clear that $\left(V^{\geq a}\right)$ is a filtration of $V$. We show:

(a) If $a \geq 1$ we have $\left.Q\right|_{V \geq a}=0$ and $V^{\geq 1-a}=\left(V^{\geq a}\right)^{\perp}$.

We use induction on $D$. For $N=0$ the result is obvious. Thus the result holds when $D \leq 1$. Now assume that $D \geq 2, N \neq 0$. Let $L, V^{\prime}, Q^{\prime}, N^{\prime}$ be as above. By the induction hypothesis we have $\left.Q^{\prime}\right|_{V_{N^{\prime}}^{\prime \geq a}}=0$ and $V_{N^{\prime}}^{\prime \geq 1-a}=\left(V^{\prime \geq a} N^{\prime}\right)^{\perp^{\prime}}$ with $\perp^{\prime}$ relative to $V^{\prime}$. It follows that $\left.Q\right|_{V \geq a}=0$ and $V^{\geq 1-a}=\left(V^{\geq a}\right)^{\perp}$ for $a \in[1, e-1]$. For $a \geq e$ the result is again true since $\left.Q\right|_{\{0\}}=0$ and $\{0\}^{\perp}=V$. This proves (a).

We see that $V^{*}=\left(V^{\geq a}\right)$ is a $Q$-filtration of $V$. Clearly, $V^{\geq a}$ is the same as the subspace $V_{\geq a}^{N}$ defined in terms of the nilpotent endomorphism $N: V \rightarrow V$ (without reference to $Q$ ) in [L2, 2.3, 2.4]. It follows that $N V^{\geq a} \subset V^{\geq a+2}$ for any $a \in \mathbf{Z}$. For any $a \in \mathbf{Z}$ we set $\bar{V}^{a}=V^{\geq a} / V^{\geq a+1}$. We see that $N$ induces a linear $\operatorname{map} \bar{N}: \bar{V}^{a} \rightarrow \bar{V}^{a+2}$. From [L2, 2.3] we see that for any $a \geq 0, \bar{N}^{a}: \bar{V}^{-a} \rightarrow \bar{V}^{-a}$ is an isomorphism. It follows that $N \in E_{*}^{\geq 2} V^{*}$. Conversely if $X^{*}$ is a $Q$-filtration of $V$ such that $N \in E_{*}^{\geq 2} X^{*}$ we see that for any $a \geq 0$ the kernel $\mathcal{K}_{a}$ of the map $\mathrm{gr}^{-a} X^{*} \rightarrow \mathrm{gr}^{a} X^{*}$ induced by $N^{a}$ is the radical of the symmetric bilinear form attached to a nondegenerate quadratic form on $\mathrm{gr}^{-a} X^{*}$; since $p \neq 2$ it follows that $\mathcal{K}_{a}=0$. Hence the map $\operatorname{gr}^{-a} X^{*} \rightarrow \operatorname{gr}^{a} X^{*}$ induced by $N^{a}$ is an isomorphism. Using $[\mathrm{L} 2,2.4]$ it follows that $X^{*}=V^{*}$. Thus $1.7(\mathrm{a})$ holds.

\section{On unipotent Conjugacy Classes in $S O_{Q}(p=2)$}

4.1. In this section we assume that $\mathbf{k}$ is algebraically closed. Moreover in this and the next subsection we assume that $p=2$. Assume that $D \geq 2$. Let $\phi \in \tilde{\mathcal{F}}_{1}^{D}$. (See 1.6.) Thus $\phi=\left(f_{a}\right)$ where $f_{0}>0$. Let

$\mathfrak{X}_{\phi}=\left\{i \in \mathbf{Z}_{\geq 1}^{\prime} ; i=f_{a}\right.$ for some $\left.a \in \mathbf{Z}_{\geq 0}^{\prime \prime}\right\}$.

this is a finite set. Let $\mathcal{E}_{\phi}$ be the set of all subsets of $\mathfrak{X}_{\phi} \times \mathfrak{X}_{\phi}$ which are equivalence relations on $\mathfrak{X}_{\phi}$. To any $X^{*} \in \overline{\mathcal{Y}}_{\phi}$ (see 1.6) and any $\bar{N} \in E_{*}^{2} \mathrm{gr}^{*} X^{*}$ we associate an element $S \in \mathcal{E}_{\phi}$ as follows. For any $i \in \mathfrak{X}_{\phi}$ let $Z_{i}$ be the subspace of $\operatorname{gr}^{0} X^{*}$ 
given by the image of the imbedding $\operatorname{gr}^{-a / 2} X^{*} \rightarrow \operatorname{gr}^{0} X^{*}$ induced by $\bar{N}^{a / 2}$ for some/any $a \in \mathbf{Z}_{\geq 0}^{\prime \prime}$ such that $i=f_{a}$; the natural symplectic form $\langle$,$\rangle on \operatorname{gr}^{0} X^{*}$ restricts to a symplectic form on $Z_{i}$ with 1-dimensional radical denoted by $L_{i}$. By definition, $S=\left\{(i, j) \in \mathfrak{X}_{\phi} \times \mathfrak{X}_{\phi} ; L_{i}=L_{j}\right\}$. For $X^{*} \in \overline{\mathcal{Y}}_{\phi}$ and $S \in \mathcal{E}_{\phi}$ let $E_{*}^{2} \mathrm{gr}^{*} X_{S}^{*}$ be the set of all $\bar{N} \in E_{*}^{2} \mathrm{gr}^{*} X^{*}$ such that $\left(X^{*}, \bar{N}\right)$ give rise to $S$ as above. Let $E^{\geq 2} X_{S}^{*}=\Phi^{-1}\left(E_{*}^{2} \operatorname{gr}^{*} X_{S}^{*}\right)$ where $\Phi$ is as in 1.5. We thus obtain a partition

$E_{*}^{\geq 2} X^{*}=\sqcup_{S \in \mathcal{E}_{\phi}} E_{*}^{\geq 2} X_{S}^{*}$

into finitely many locally closed subvarieties. For $S \in \mathcal{E}_{\phi}$ let

$$
\Xi_{\phi}^{S}=\left\{g \in S O_{Q} ; g \text { unipotent } g-1 \in E_{*}^{\geq 2} X_{S}^{*}\right\} \text {. }
$$

Hence $\Xi_{\phi}$ (see 1.7) is partitioned as $\Xi_{\phi}=\sqcup_{S \in \mathcal{E}_{\phi}} \Xi_{\phi}^{S}$. Note that each $\Xi_{\phi}^{S}$ is stable under conjugation by $S O_{Q}$. One can show that the sets $\Xi_{\phi}^{S}$ with $\phi, S$ as above (together with the sets $\Xi_{\phi}$ with $\phi \in \mathcal{F}^{D}-\tilde{\mathcal{F}}_{1}^{D}$ in the case where $D \in \mathbf{Z}^{\prime \prime}$ ) are exactly the unipotent conjugacy classes in $S O_{Q}$. A quite different classification of unipotent conjugacy classes in $S O_{Q}$ is given in [W].

4.2. Let $\mathcal{N}_{Q}$ be the set of nilpotent elements $\nabla \in \operatorname{End}(V)$ such that $\langle x, \nabla y\rangle+$ $\langle\nabla x, y\rangle=0$ for all $x, y$ in $V$ and $\langle x, \nabla x\rangle=0$ for all $x \in V$. We can view $\mathcal{N}_{Q}$ as the set of nilpotent elements in the Lie algebra of $S O_{Q}$. Note that $S O_{Q}$ acts by conjugation on $\mathcal{N}_{Q}$.

Let $u$ be a unipotent element in $S O_{Q}$. We associate to $u$ a $S O_{Q}$-conjugacy class in $\mathcal{N}_{Q}$ as follows. Let $N=u-1 \in \mathcal{M}_{Q}$. Let $X^{*}=V_{N}^{*}$. Then $N \in E^{\geq 2} X^{*}$ and $\bar{N} \in E^{2} \operatorname{gr} X^{*}$ is defined as in 1.5. Let $\bar{Q}$ be the quadratic form on $\operatorname{gr} X^{*}$ defined in 1.5. We have $\bar{N} \in \mathcal{N}_{\bar{Q}}$. Note that if $D \in \mathbf{Z}^{\prime \prime}$ then the set of connected components of $\mathcal{J}_{Q}$ and that of $\mathcal{J}_{\bar{Q}}$ may be naturally identified. We can find an isomorphism of vector spaces $\operatorname{gr} X^{*} \stackrel{\sim}{\longrightarrow} V$ which carries $\bar{Q}$ to $Q$ and (when $D \in \mathbf{Z}^{\prime \prime}$ ) induces the identity map from the set of connected components of $\mathcal{J}_{\bar{Q}}$ to that of $\mathcal{J}_{Q}$. This isomorphism carries $\bar{N}$ to an element $\nabla \in \mathcal{N}_{Q}$ whose $S O_{Q}$-orbit is independent of the choice of isomorphism. Note that $1+N \mapsto \nabla$ defines a map

$$
\begin{aligned}
& \left\{S O_{Q}-\text { conjugacy classes of unipotent elements in } S O_{Q}\right\} \\
& \rightarrow\left\{S O_{Q}-\text { conjugacy classes of nilpotent elements in Lie } S O_{Q}\right\} .
\end{aligned}
$$

One can show that this map is injective; it is not in general surjective.

4.3. The map 4.2(a) makes sense also in the more general framework of [L2]. Assume that $p>1$. Let $G$ be as in $[\mathrm{L} 2,0.1]$. We assume that property $\mathfrak{P}_{1}$ in $[\mathrm{L} 2,1.1]$ holds for $G$. Let $u$ be a unipotent element in $G$. By $\mathfrak{P}_{1}$ we can find a unique sequence $\Delta=\left(G_{0}^{\Delta} \supset G_{1}^{\Delta} \supset G_{2}^{\Delta} \supset \ldots\right)$ in $D_{G}$ such that $u \in X^{\Delta}$ (notation of $\left[\right.$ L2, 1.1]). In particular we have $u \in G_{2}^{\Delta}$. Let $\mathfrak{g}=\operatorname{Lie} G, \mathfrak{g}_{n}^{\Delta}=$ Lie $G_{n}^{\Delta}$. Let $\bar{u}$ be the image of $u$ in $G_{2}^{\Delta} / G_{3}^{\Delta}$. Since $G_{2}^{\Delta} / G_{3}^{\Delta}$ is a connected commutative unipotent group in which the $p$-th power of any element is 1 we see that it is canonically isomorphic to its Lie algebra $\mathfrak{g}_{2}^{\Delta} / \mathfrak{g}_{3}^{\Delta}$. Hence $\bar{u}$ can be identified with an element of $\mathfrak{g}_{2}^{\Delta} / \mathfrak{g}_{3}^{\Delta}$. By definition there exists a homomorphism of algebraic groups $h: \mathbf{k}^{*} \rightarrow G$ 
such that if we denote by $\mathfrak{g}^{n}$ the $n$-eigenspace of the action $x \mapsto \operatorname{Ad}(h(x))$ of $\mathbf{k}^{*}$ on $\mathfrak{g}(n \in \mathbf{Z})$ we have $\mathfrak{g}_{n}^{\Delta}=\mathfrak{g}^{n} \oplus \mathfrak{g}^{n+1} \oplus \ldots$ for any $n \geq 0$; moreover $h$ is unique up to $G_{0}^{\Delta}$-conjugacy. Using the decomposition $\mathfrak{g}_{2}^{\Delta}=\mathfrak{g}^{2} \oplus \mathfrak{g}_{3}^{\Delta}$ we can identify $\mathfrak{g}_{2}^{\Delta} / \mathfrak{g}_{3}^{\Delta}$ with $\mathfrak{g}^{2}$ and we can view $\bar{u}$ as an element of $\mathfrak{g}^{2}$ hence as a nilpotent element of $\mathfrak{g}$. This element is well defined up to $G_{0}^{\Delta}$-conjugacy. This defines a map

$\{$ unipotent $G$-conjugacy classes in $G\} \rightarrow\{$ nilpotent $G$-conjugacy classes in $\mathfrak{g}$ \}.

\section{REFERENCES}

[K] B.Kostant, The principal three dimensional subgroup and the Betti numbers of a complex simple Lie group, Amer.J.Math. 81 (1959), 973-1032.

[L1] G.Lusztig, Notes on unipotent classes, Asian J.Math. 1 (1997), 194-207.

[L2] G.Lusztig, Unipotent elements in small characteristic, Transfor.Groups 10 (2005), 449-487.

[W] G.E.Wall, On the conjugacy classes in the unitary, symplectic and orthogonal groups, J.Austral.Math.Soc. 3 (1963), 1-62.

Department of Mathematics, M.I.T., Cambridge, MA 02139 